\title{
CLASSES CARACTÉRISTIQUES NON TRIVIALES DE SOUS-FEUILLETAGES LOCALEMENT HOMOGÈNES
}

\author{
Par Demetrio Domínguez
}

\section{$\S 1$. Introduction.}

Nous reprenons les notations de [5]. Plus particulièrement, nous considérons un sous-feuilletage localement homogène $\left(F_{1}, F_{2}\right)=\left(F_{G_{1}}, F_{G_{2}}\right)$ de codimension $\left(q_{1}, q_{2}\right)=\left(\operatorname{dim} \bar{g} / g_{1}, \operatorname{dim} \bar{g} / g_{2}\right)$ sur une variété différentiable $M=\Gamma \backslash \bar{G} / H$ de dimension $n=\operatorname{dim} \bar{g} / h$, où $H \subset G_{2} \subset G_{1} \subset \bar{G}$ désignent des groupes de Lie, $h \subset g_{2} \subset g_{1} \subset \bar{g}$ leurs algèbres de Lie, $H$ étant fermé dans $\bar{G}$, et $\Gamma \subset \bar{G}$ un sous-groupe discret opérant de manière proprement discontinue et sans points fixes sur $\bar{G} / H$. Le sous-feuilletage $\left(F_{1}, F_{2}\right)$ sur $M$ est induit par le sous-feuilletage sur $\bar{G}$ determiné par les orbites des opérations à droite de $G_{1}$ et $G_{2}$. En particulier, $d=q_{2}-q_{1}$ $=\operatorname{dim} g_{1} / g_{2}$.

Roussarie [7], Kamber-Tondeur [9] et Yamato [15] construisent des feuilletages localement homogènes ayant des classes caractéristiques secondaires ou exotiques non nulles. Cordero-Masa [4] et Carballés [3] ne donnent aucun exemple de sous-feuilletages $\left(F_{1}, F_{2}\right)$ sur $M$, admettant des classes caractéristiques secondaires ou exotiques non nulles qui ne peuvent pas s'obtenir en considérant séparément les feuilletages de la paire.

Dans ce travail, nous décrivons le calcul de l'homomorphisme caractéristique pour les sous-feuilletages localement homogènes, en utilisant des techniques analogues à celles de Kamber-Tondeur [9], [10] et [11]. Pour appliquer les résultats, nous donnons quelques exemples de sous-feuilletages localement homogènes $\left(F_{1}, F_{2}\right)$ sur $M$, ayant des classes caractéristiques secondaires non nulles qui n'appartiennent pas à la sous-algèbre de $H_{D R}(M)$ engendrée par les classes caractéristiques des feuilletages de la paire.

Le $\S 2$ montre que le fibré normal $\nu\left(F_{1}, F_{2}\right)=Q_{1} \oplus Q_{0}=\left(T M / F_{1}\right) \oplus\left(F_{1} / F_{2}\right)$ d'un sous-feuilletage localement homogène $\left(F_{1}, F_{2}\right)=\left(F_{G_{1}}, F_{G_{2}}\right)$ de codimension $\left(q_{1}, q_{2}\right)$ $=\left(q_{1}, q_{1}+d\right)$ sur $M=\Gamma \backslash \bar{G} / H$ est associé au fibré principal $\left(F_{1}, F_{2}\right)$-feuilleté localement homogène

$$
P=P_{1}+P_{2}=\Gamma \backslash \bar{G} \times{ }_{H} G_{1}+\Gamma \backslash \bar{G} \times{ }_{H} G_{2}=\Gamma \backslash \bar{G} \times{ }_{H}\left(G_{1} \times G_{2}\right) \rightarrow M
$$

par la représentation d'isotropie

$$
\rho=\left(\rho_{1}, \rho_{0}\right): G_{1} \times G_{2} \rightarrow G L\left(\bar{g} / g_{1}\right) \times G L\left(g_{1} / g_{2}\right) \cong G L\left(q_{1}\right) \times G L(d),
$$

Received September 28, 1987 
et que la structure $\left(F_{1}, F_{2}\right)$-feuilletée induite par celle de $P=P_{1}+P_{2}$ dans $Q_{1} \oplus Q_{0}$ coïncide avec la connexion partielle de Bott somme. Par suite, si $\rho(H \times H) \subset O\left(q_{1}\right) \times O(d)$, l'homomorphisme caractéristique $\Delta_{*}$ du sous-feuilletage $\left(F_{1}, F_{2}\right)$ se factorise de la manière suivante:

$$
\Delta_{*}: H\left(W O_{I}\right) \stackrel{w(d \rho)^{*}}{\longrightarrow} H\left(W\left(g_{1} \oplus g_{2}, H \times H\right)_{I}\right) \stackrel{\Delta(\theta)_{*}}{\longrightarrow} H(\bar{g}, H) \stackrel{\gamma_{*}}{\longrightarrow} H_{D R}(M),
$$

$\Delta_{*}(P)=\gamma_{*}{ }^{\circ} \Delta(\theta)_{*}$ étant l'homomorphisme caractéristique généralisé du fibré principal $\left(F_{1}, F_{2}\right)$-feuilleté localement homogène $P$, où $\theta=\theta_{1}+\theta_{2}: \bar{g} \rightarrow g_{1} \oplus g_{2}$ désigne l'application linéaire déterminée par une certaine connexion adaptée somme $\omega=\omega_{1}+\omega_{2}$ sur $P=P_{1}+P_{2}$, et où $\gamma:\left(\Lambda \bar{g}^{*}\right)_{H} \rightarrow \Omega(M)$ est l'inclusion canonique. Ces résultats réduisent le calcul de l'homomorphisme caractéristique d'un sousfeuilletage localement homogène à un calcul purement algébrique qui peut se faire moyennant certaines hypothèses avec les méthodes des paragraphes 3,4 et 5 de ce travail.

$\mathrm{Au} \S 3$ on considère quelques fibrés plats de la forme $\bar{P}=\Gamma \backslash \bar{G} \times{ }_{H} \bar{G} \rightarrow$ $M=\Gamma \backslash \bar{G} / H$ dont l'homomorphisme caractéristique généralisé $\Delta_{*}(\bar{P})=\gamma_{*}: H(\bar{g}, H)$ $\rightarrow H_{D R}(M)$ est injectif.

Le $\S 4$ étudie des classes de cohomologie dans $H\left(W\left(g_{1} \oplus g_{2}, H_{1} \times H_{2}\right)_{I}\right)$ et contient le calcul de l'homomorphisme $\Delta(\theta)_{*}: H\left(W\left(g_{1} \oplus g_{2}, H \times H\right)_{I}\right) \rightarrow H(\bar{g}, H)$ pour certains cas particuliers.

Le $\S 5$ réduit, pour certaines paires de groupes de Lie, le calcul de l'homomorphisme $W(d \rho)^{*}: H\left(W\left(g^{\prime}, H^{\prime}\right)_{I}\right) \rightarrow H\left(W(g, H)_{I}\right)$ à celui d'un homomorphisme (d'algèbres graduées) $\hat{\rho}^{*}: H\left(\hat{A}\left(W\left(g^{\prime}\right)_{I}\right)\right) \rightarrow H\left(\hat{A}\left(W(g)_{I}\right)\right)$. Ces résultats s'appliquent en particulier au problème du prolongement de feuilletages.

Enfin, au $\S 6$ on donne, pour appliquer les résultats des paragraphes précédents, quelques exemples de sous-feuilletages localement homogènes $\left(F_{1}, F_{2}\right)$ sur $M$, ayant des classes caractéristiques secondaires non nulles qui n'appartiennent pas à la sous-algèbre de $H_{D R}(M)$ engendrée par les classes caractéristiques des feuilletages de la paire. L'un de ces exemples peut être considéré comme une généralisation de l'exemple donné par Roussarie dans [7]. De même, on interprète géométriquement ces nouveaux invariants.

J'exprime ma gratitude et remercie à X. M. Masa qui a présidé à l'élaboration de ce travail.

\section{§. Classes caractéristiques des sous-feuilletages localement homogènes.}

Dans ce paragraphe, en utilisant des techniques similaires à celles de Kamber-Tondeur [10], nous montrons que l'homomorphisme caractéristique d'un sous-feuilletage localement homogène est le composé de trois homomorphismes qui réduisent le calcul d'un tel homomorphisme à un calcul purement algébrique.

Soient $H \subset G_{2} \subset G_{1} \subset \bar{G}$ des groupes de Lie, $h \subset g_{2} \subset g_{1} \subset \bar{g}$ leurs algèbres de Lie. Supposons que $H$ soit fermé dans $\bar{G}$. Soit $\Gamma \subset \bar{G}$ un sous-groupe discret opérant de manière proprement discontinue et sans points fixes sur $\bar{G} / H$; par 
conséquent, l'espace localement homogène $M=\Gamma \backslash \bar{G} / H$ est une variété différentiable de dimension $n=\operatorname{dim} \bar{g} / h$. Pour chaque $i=1,2$, considérons le fibré principal feuilleté localement homogène $P_{i}=\Gamma \backslash \bar{G} \times{ }_{H} G_{i} \rightarrow M$, où le feuilletage sur $P_{i}$ est induit par les orbites de l'action diagonale de $G_{i}$ sur $\bar{G} \times G_{i}$, et où le feuilletage localement homogène $F_{i}=F_{G_{i}}$ de codimension $q_{i}=\operatorname{dim} \bar{g} / g_{i}$ sur $M$ est induit par le feuilletage sur $\bar{G}$ défini par l'opération à droite de $G_{i}$. Puisque $G_{2} \subset G_{1}$, on vérifie que $\left(F_{1}, F_{2}\right)$ est un sous-feuilletage de codimension $\left(q_{1}, q_{2}\right)$ sur $M$ et que

$$
P=P_{1}+P_{2}=\Gamma \backslash \bar{G} \times{ }_{H} G_{1}+\Gamma \backslash \bar{G} \times{ }_{H} G_{2}=\Gamma \backslash \bar{G} \times{ }_{H}\left(G_{1} \times G_{2}\right) \rightarrow M
$$

est un fibré principal $\left(F_{1}, F_{2}\right)$-feuilleté de groupe structural $G_{1} \times G_{2}$. En particulier, on a donc $d=q_{2}-q_{1}=\operatorname{dim} g_{1} / g_{2}$.

DÉFinition 2.1. Un tel fibré principal $\left(F_{1}, F_{2}\right)$-feuilleté $P=P_{1}+P_{2}$ sera appelé un fibré principal $\left(F_{1}, F_{2}\right)$-feuilleté localement homogène. Un tel sous-feuilletage $\left(F_{1}, F_{2}\right)$ sera appelé un sous-feuilletage localement homogène. Si $\Gamma=\{e\}$, on dira simplement que $P=P_{1}+P_{2}=\bar{G} \times{ }_{H}\left(G_{1} \times G_{2}\right) \rightarrow M=\bar{G} / H$ (resp. $\left(F_{1}, F_{2}\right)=\left(F_{G_{1}}, F_{G_{2}}\right)$ ) est un fibré principal $\left(F_{1}, F_{2}\right)$-feuilleté homogène (resp. un sous-feuilletage homogène).

Remarque. Soit $F=F_{G}$ un feuilletage localement homogène sur $M=\Gamma \backslash \bar{G} / H$ considéré comme un sous-feuilletage $\left(F_{1}, F_{2}\right)$. On a alors: (i) $F_{1}=F_{2}=F$ pour $G_{1}=G_{2}=G$; (ii) $F_{1}=T M$ et $F_{2}=F$ pour $G_{1}=\bar{G}$ et $G_{2}=G$; (iii) $F_{1}=F$ et $F_{2}=0$ pour $G_{1}=G$ et $G_{2}=H$.

Soit $\left(F_{1}, F_{2}\right)=\left(F_{G_{1}}, F_{G_{2}}\right)$ un sous-feuilletage localement homogène de codimension $\left(q_{1}, q_{2}\right)$ sur $M=\Gamma \backslash \bar{G} / H$, et soit $d=q_{2}-q_{1} \geqq 0$. Considérons le fibré normal $Q_{1} \oplus Q_{0}=\left(T M / F_{1}\right) \oplus\left(F_{1} / F_{2}\right)$ (resp. $\left.Q_{2}=T M / F_{2}\right)$ de $\left(F_{1}, F_{2}\right)$ (resp. de $F_{2}$ ). Nous obtenons alors le résultat suivant.

LEMme 2.2. Le fibré normal $Q_{1} \oplus Q_{0}$ de $\left(F_{1}, F_{2}\right)$ est associé au fibré principal $\left(F_{1}, F_{2}\right)$-feuilleté localement homogène $P=P_{1}+P_{2}=\Gamma \backslash \bar{G} \times{ }_{H} G_{1}+\Gamma \backslash \bar{G} \times{ }_{H} G_{2}=$ $\Gamma \backslash \bar{G} \times{ }_{H}\left(G_{1} \times G_{2}\right)$ par la représentation d'isotropie

$$
\rho=\left(\rho_{1}, \rho_{0}\right): G_{1} \times G_{2} \rightarrow G L\left(\bar{g} / g_{1}\right) \times G L\left(g_{1} / g_{2}\right) \cong G L\left(q_{1}\right) \times G L(d) .
$$

De plus, la structure $\left(F_{1}, F_{2}\right)$-feuilletée induite par celle de $P=P_{1}+P_{2}$ dans $Q_{1} \oplus Q_{0}$ coïncide avec la connexion partielle de Bott somme.

Démonstration. En effet, à la suite exacte de $H$-modules

$$
0 \rightarrow g_{2} / h \rightarrow g_{1} / h \rightarrow m_{0}=g_{1} / g_{2} \rightarrow 0
$$

correspond la suite exacte de fibrés vectoriels

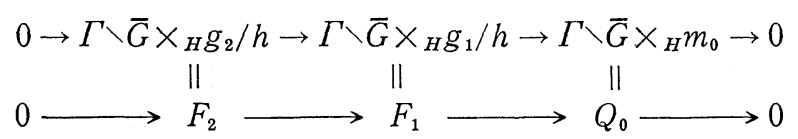


associés au $H$-fibré principal $\Gamma \backslash \bar{G} \rightarrow M$. D'après Kamber-Tondeur [10], la connexion partielle plate induite par celle de $P_{i}, i=1,2$, dans $Q_{i}=\Gamma \backslash \bar{G} \times{ }_{H} m_{i} \cong$ $P_{i} \times{ }_{G_{i}} m_{i}$ coïncide avec la connexion partielle de Bott, où $m_{i}=\bar{g} / g_{i}$. Il en résulte que la structure $F_{2}$-feuilletée induite par celle de $P_{2}$ dans $Q_{0}=\Gamma \backslash \bar{G} \times{ }_{H} m_{0} \cong P_{2} \times_{G_{2}} m_{0}$ coïncide avec la connexion partielle de Bott (donnée dans [4]), d'où le lemme.

Il est clair que la suite exacte de $G_{2}$-modules

$$
0 \rightarrow m_{0} \rightarrow m_{2} \rightarrow m_{1} \rightarrow 0
$$

conduit à la suite exacte de fibrés vectoriels

$$
0 \rightarrow Q_{0} \cong P_{2} \times_{G_{2}} m_{0} \rightarrow Q_{2} \cong P_{2} \times_{G_{2}} m_{2} \rightarrow Q_{1} \cong P_{2} \times_{G_{2}} m_{1} \cong P_{1} \times_{G_{1}} m_{1} \rightarrow 0 .
$$

Par conséquent, la structure $F_{2}$-feuilletée canonique de $Q_{1}$ est induite par la connexion partielle plate de $P_{2}$.

On en déduit le

Corollaire 2.3. S'il existe une scission $G_{2}$-équivariante $\theta_{1}: \bar{g} \rightarrow g_{1}$ de la suite exacte de $G_{1}$-modules $0 \rightarrow g_{1} \rightarrow \bar{g} \rightarrow m_{1}=\bar{g} / g_{1} \rightarrow 0$, alors $Q_{2}$ et $Q_{1} \oplus Q_{0}$ sont isomorphes comme fibrés vectoriels $F_{2}$-feuilletés.

Pour simplifier le calcul de l'homomorphisme caractéristique d'un sous-feuilletage localement homogène, supposons que $\rho(H \times H) \subset O\left(q_{1}\right) \times O(d)$ et que, pour chaque $i=1,2$, il existe une scission $H$-équivariante $\theta_{i}: \bar{g} \rightarrow g_{i}$ de la suite exacte de $G_{\imath}$-modules

$$
0 \rightarrow g_{i} \rightarrow \bar{g} \rightarrow m_{i}=\bar{g} / g_{i} \rightarrow 0 .
$$

Ces conditions sont évidemment vérifiées si $H$ est un sous-groupe compact de $\bar{G}$. D'autre part, on considère la section $s: M \rightarrow P /(H \times H)$ de la projection canonique $\hat{\pi}: P /(H \times H) \rightarrow M$, induite par la section canonique $\bar{G} \rightarrow \bar{G} \times(\{e\} \times\{e\})$ de $\bar{G} \times\left(G_{1} \times G_{2}\right) \rightarrow \bar{G}$. Alors, d'après Kamber-Tondeur [10], le $H-D G$-homomorphisme $\Delta\left(\theta_{i}\right): W\left(g_{i}\right) \rightarrow \Lambda \bar{g}^{*}, \quad i=1,2$, induit par $\theta_{i}$ satisfait $\Delta\left(\theta_{i}\right) F^{2 p} W\left(g_{i}\right) \subset$ $F^{p}\left(g_{i}\right) \Lambda \bar{g}^{*}=\Lambda^{p} m_{i}^{*} \cdot \Lambda \bar{g}^{*}$ pour $p \geqq 0$; par conséquent, $F^{2\left(q_{i+1}\right)} W\left(g_{i}\right) \subset \operatorname{Ker} \Delta\left(\theta_{i}\right)$. Si, de plus, $\theta_{i}, i=1,2$, est $G_{i}$-équivariante, $\Delta\left(\theta_{i}\right)$ est un $G_{i}-D G$-homomorphisme satisfaisant $\Delta\left(\theta_{i}\right) F^{2 p} W\left(g_{i}\right) \subset F^{2 p}\left(g_{i}\right) \Lambda \bar{g}^{*}$ pour $p \geqq 0$; par suite, on a $F^{2\left(\left[q_{i} / 2\right]+1\right)} W\left(g_{i}\right)$ $\subset \operatorname{Ker} \Delta\left(\theta_{i}\right)$.

On désigne maintenant par $\theta=\theta_{1}+\theta_{2}$ l'application linéaire composée $\left(\theta_{1}, \theta_{2}\right) \circ \Delta: \bar{g} \rightarrow \bar{g} \oplus \bar{g} \rightarrow g_{1} \oplus g_{2}$, où $\Delta$ est l'application diagonale. Puisque le $H-D G$-homomorphisme $\Delta(\theta): W\left(g_{1} \oplus g_{2}\right) \cong W\left(g_{1}\right) \otimes W\left(g_{2}\right) \rightarrow \Lambda \bar{g}^{*}$ induit par $\theta$ est donné par la composition $\mu \circ\left(\Delta\left(\theta_{1}\right) \otimes \Delta\left(\theta_{2}\right)\right)$, où $\mu$ désigne la multiplication de $\Lambda \bar{g}^{*}$, on obtient alors $\Delta(\theta)^{\prime} F^{2 p} W\left(g_{1} \oplus g_{2}\right) \subset F^{p}\left(g_{1}\right) \Lambda \bar{g}^{*}$ et $\Delta(\theta) F^{2 p} W\left(g_{1} \oplus g_{2}\right) \subset$ $F^{p}\left(g_{2}\right) \Lambda \bar{g}^{*}$ pour $p \geqq 0$. Par conséquent, $I==^{\prime} F^{2\left(q_{1}+1\right)} W\left(g_{1} \oplus g_{2}\right)+F^{2\left(q_{2}+1\right)} W\left(g_{1} \oplus g_{2}\right)$ $\subset \operatorname{Ker} \Delta(\theta)$. Si, pour chaque $i=1,2, \theta_{i}$ est $G_{i}$-équivariante, alors on vérifie $\Delta(\theta)^{\prime} F^{2 p} W\left(g_{1} \oplus g_{2}\right) \subset F^{2 p}\left(g_{1}\right) \Lambda \bar{g}^{*}$ et $\Delta(\theta) F^{2 p} W\left(g_{1} \oplus g_{2}\right) \subset F^{2 p}\left(g_{2}\right) \Lambda \bar{g}^{*}$ pour $p \geqq 0$; par suite, $I^{\prime \prime}=^{\prime} F^{2\left(\left[\left(q_{1}+1\right) / 2\right]+1\right)} W\left(g_{1} \oplus g_{2}\right)+F^{2\left(\left[\left(q_{2}+1\right) / 2\right]+1\right)} W\left(g_{1} \oplus g_{2}\right) \subset \operatorname{Ker} \Delta(\theta)$. 
D'autre part, considérons la connexion adaptée somme $\omega=\omega_{1}+\omega_{2}$ sur $P=P_{1}+P_{2}=\Gamma \backslash \bar{G} \times{ }_{H}\left(G_{1} \times G_{2}\right)$, déterminée par $\theta=\theta_{1}+\theta_{2}$. Il est clair que $\omega$ est une connexion basique somme sur $P$ si, pour chaque $i=1,2, \theta_{i}$ est $G_{i}$-équivariante. Alors, par un raisonnement analogue à celui qui a conduit au Théorème 3.7 dans [10], on obtient le résultat suivant.

LEMME 2.4. L'homomorphisme caractéristique généralisé $\Delta_{*}(P)$ du fibré principal $\left(F_{1}, F_{2}\right)$-feuilleté localement homogène $P=P_{1}+P_{2}$ se factorise de la manière suivante:

$$
\Delta_{*}(P): H\left(W\left(g_{1} \oplus g_{2}, H \times H\right)_{I}\right) \stackrel{\Delta(\theta)_{*}}{\longrightarrow} H(\bar{g}, H) \stackrel{\gamma_{*}}{\longrightarrow} H_{D R}(M),
$$

où $\gamma:\left(\Lambda \bar{g}^{*}\right)_{H} \rightarrow \Omega(M)$ est l'inclusion canonique. Si, de plus, $\theta_{i}$ est $G_{i}$-équivariante pour $i=1,2$, alors l'idéal $I$ est remplacé par $I^{\prime \prime}$.

On déduit des Lemmes 2.2 et 2.4 le

THÉORÈME 2.5. L'homomorphisme caractéristique $\Delta_{*}$ du sous-feuilletage localement homogène $\left(F_{1}, F_{2}\right)=\left(F_{G_{1}}, F_{G_{2}}\right)$ de codimension $\left(q_{1}, q_{2}\right)$ sur $M=\Gamma \backslash \bar{G} / H$ est donné par la composition

$$
\Delta_{*}: H\left(W O_{I}\right) \stackrel{w(d \rho)^{*}}{\longrightarrow} H\left(W\left(g_{1} \oplus g_{2}, H \times H\right)_{I}\right) \stackrel{\Delta(\theta)_{*}}{\longrightarrow} H(\bar{g}, H) \stackrel{\gamma_{*}}{\longrightarrow} H_{D R}(M) .
$$

Si $\theta_{i}, i=1,2$, est $G_{i}$-équivariante, alors $I$ est remplacé par $I^{\prime \prime}$.

Remarques. 1) Il est clair que, pour les homomorphismes caractéristiques généralisés $\Delta_{*}\left(P_{1}\right), \Delta_{*}\left(P_{2}\right), \Delta_{* F_{2}}(P)$ (de $P$ considéré comme un fibré principal $F_{2}$-feuilleté), $\Delta_{*}\left(Q_{1}\right), \Delta_{*}\left(Q_{0}\right), \Delta_{* F_{2}}$ (de $Q_{1} \oplus Q_{0}$ considéré comme un fibré vectoriel $F_{2}$-feuilleté) et $\Delta_{*}\left(Q_{2}\right)$, on a alors les factorisations suivantes:

$$
\begin{aligned}
& \Delta_{*}\left(P_{i}\right)=\gamma_{*} \circ \Delta\left(\theta_{i}\right)_{*}=\Delta_{*}(P) \circ W\left(d p_{i}\right)^{*}: H\left(W\left(g_{i}, H\right)_{q_{2}}\right) \rightarrow H_{D R}(M) \quad \text { pour } i=1,2 ; \\
& \Delta_{* F_{2}}(P)=\gamma_{*} \circ \Delta(\theta)_{* F_{2}}=\Delta_{*}(P) \circ p_{*}: H\left(W\left(g_{1} \oplus g_{2}, H \times H\right)_{q_{2}}\right) \rightarrow H_{D R}(M) ; \\
& \Delta_{*}\left(Q_{1}\right)=\Delta_{*}\left(P_{1}\right) \circ W\left(d \rho_{1}\right)^{*}=\Delta_{*} \circ W\left(d p_{1}^{\prime}\right)^{*}: H\left(W O_{q_{1}}\right) \rightarrow H_{D R}(M) ; \\
& \Delta_{*}\left(Q_{0}\right)=\Delta_{*}\left(P_{2}\right) \circ W\left(d \rho_{0}\right)^{*}=\Delta_{*} \circ W\left(d p_{2}^{\prime}\right)^{*}: H\left(W(g l(d), O(d))_{q_{2}}\right) \rightarrow H_{D R}(M) ; \\
& \Delta_{* F_{2}}=\Delta_{* F_{2}}(P) \circ W(d \rho)^{*}=\Delta_{*} \circ p_{*}^{\prime}: H\left(W\left(g l\left(q_{1}\right) \oplus g l(d), O\left(q_{1}\right) \times O(d)\right)_{q_{2}}\right) \rightarrow H_{D R}(M) ; \\
& \Delta_{*}\left(Q_{2}\right)=\Delta_{*}\left(P_{2}\right) \circ W\left(d \rho_{2}\right)^{*}=\Delta_{*} \circ p_{*}^{\prime} \circ W\left(d \rho^{\prime}\right)^{*}: H\left(W O_{q_{2}}\right) \rightarrow H_{D R}(M),
\end{aligned}
$$

où $p, p^{\prime}, p_{i}$ et $p_{i}^{\prime}, i=1,2$, sont les homomorphismes canoniques, où $\rho_{2}: G_{2} \rightarrow$ $G L\left(\bar{g} / g_{2}\right) \cong G L\left(q_{2}\right)$ est la représentation d'isotropie, et où $\rho^{\prime}: G L\left(q_{1}\right) \times G L(d) \rightarrow$ $G L\left(q_{2}\right)$ est l'inclusion canonique. De même, si $\theta_{i}$, $i=1,2$, est $G_{i}$-équivariante, alors, pour tronquer les algèbres de Weil précédentes, $q_{i}, i=1,2$ (resp. I) est remplacé par $\left[\left(q_{i}+1\right) / 2\right]$ (resp. par $\left.I^{\prime \prime}\right)$.

2) On peut aussi considérer les cas particuliers suivants: 
i ) Pour $G_{1}$ et $G_{2}$ connexes, on vérifie que $\left(F_{1}, F_{2}\right)=\left(F_{G_{1}}, F_{G_{2}}\right)$ est un sousfeuilletage à fibré normal orientable et que la structure $\left(F_{1}, F_{2}\right)$-feuilletée du fibré $P^{\prime}=L\left(Q_{1}\right)+L\left(Q_{0}\right)$ des repères transverses à $\left(F_{1}, F_{2}\right)$ est induite par une structure $\left(F_{1}, F_{2}\right)$-feuilletée d'une $G L^{+}\left(q_{1}\right) \times G L^{+}(d)$-réduction de $P^{\prime}$. Dans ce cas, la cohomologie $H\left(W O_{I}\right) \cong H\left(W\left(g l\left(q_{1}\right) \oplus g l(d), O\left(q_{1}\right) \times O(d)\right)_{I}\right)$ est remplacée par $H\left(W\left(g l\left(q_{1}\right) \oplus g l(d), S O\left(q_{1}\right) \times S O(d)\right)_{I}\right)$.

ii) Pour $G_{1}$ et $G_{2}$ compacts, $\left(F_{1}, F_{2}\right)$ est un sous-feuilletage riemannien; par conséquent, $H\left(W O_{I}\right)$ est remplacé par $H\left(W\left(s o\left(q_{1}\right) \oplus s o(d), H_{1} \times H_{2}\right)_{I^{\prime \prime}}\right)$, où $H_{1} \times H_{2} \subset O\left(q_{1}\right) \times O(d)$ est un sous-groupe fermé tel que $\rho(H \times H) \subset H_{1} \times H_{2}$.

iii) Pour $H=\{e\},\left(F_{1}, F_{2}\right)$ est un sous-feuilletage à fibré normal trivialisé ; par suite, $H\left(W O_{I}\right)$ est remplacé par $H\left(W_{I}\right) \cong H\left(W\left(g l\left(q_{1}\right) \oplus g l(d)\right)_{I}\right)$.

Dans le calcul de l'homomorphisme caractéristique d'un sous-feuilletage localement homogène, les résultats suivants sont intéressants.

Proposition 2.6. Il exists un DG-homomorphisme $k: W\left(g_{1} \oplus g_{2}, H \times H\right)_{I} \rightarrow$ $W\left(g_{1}, H\right)_{q_{1}}$ tel que $k \circ W\left(d p_{1}\right)=i d$ et $\Delta_{*}(P)=\Delta_{*}\left(P_{1}\right) \circ k_{*}$. Par suite, on a alors $\operatorname{Im} \Delta_{*}(P)=\operatorname{Im} \Delta_{*}\left(P_{1}\right)$. Si $\theta_{i}, i=1,2$, est $G_{2}$-équivariante, $q_{1}$ (resp. I) est remplacé par $\left[\left(q_{1}+1\right) / 2\right]$ (resp. par $\left.I^{\prime \prime}\right)$.

Démonstration. En effect, $k$ est le $D G$-homomorphisme induit par le $H$-DG-homomorphisme composé

$$
\mu \circ\left(i d \otimes k\left(\theta_{0}\right)\right): W\left(g_{1} \oplus g_{2}\right) \cong W\left(g_{1}\right) \otimes W\left(g_{2}\right) \rightarrow W\left(g_{1}\right) \otimes W\left(g_{1}\right) \rightarrow W\left(g_{1}\right),
$$

où $k\left(\theta_{0}\right)$ est l'homomorphisme de Weil de la scission $H$-équivariante $\theta_{0}=\theta_{2} \circ i_{1}$ : $g_{1} \rightarrow \bar{g} \rightarrow g_{2}$ de $0 \rightarrow g_{2} \rightarrow g_{1} \rightarrow g_{1} / g_{2} \rightarrow 0$ ( $i_{1}$ étant l'inclusion canonique), et où $\mu$ est la multiplication de $W\left(g_{1}\right)$. On achève donc la démonstration en appliquant le Lemme 4.9 dans [11] à l'homomorphisme $k\left(\theta_{0}\right)$.

On en déduit le

Corollaire 2.7. Pour que $\operatorname{Im} \Delta_{*} \neq \operatorname{Im} \Delta_{*}\left(Q_{1}\right)$, il faut que $\operatorname{Im} \Delta_{*}\left(Q_{1}\right) \neq \operatorname{Im} \Delta_{*}\left(P_{1}\right)$.

Soient $i_{0}: G_{2} \rightarrow G_{1}$ l'inclusion canonique et $\Delta: G_{2} \rightarrow G_{2} \times G_{2}$ l'application diagonale. Considérons l'homomorphisme composé $\bar{\rho}=\left(i_{0}, i d\right) \bullet \Delta: G_{2} \rightarrow G_{1} \times G_{2}$. On vérifie évidemment que la structure $F_{2}$-feuilletée canonique de $P=\Gamma \backslash \bar{G} \times{ }_{H}\left(G_{1} \times G_{2}\right)$ est la $\bar{\rho}$-extension de la structure $F_{2}$-feuilletée de $P_{2}=\Gamma \backslash \bar{G} \times{ }_{H} G_{2}$. On a ainsi démontré la

Proposition 2.8. Il existe un DG-homomorphisme $\alpha: W\left(g_{1} \oplus g_{2}, H \times H\right)_{q_{2}} \rightarrow$ $W\left(g_{2}, H\right)_{q_{2}}$ tel que $\alpha \circ W\left(d p_{2}\right)=i d$ et $\Delta_{* F_{2}}(P)=\Delta_{*}\left(P_{2}\right) \circ \alpha_{*}$. Il s'ensuit que $\operatorname{Im} \Delta_{* F_{2}}(P)$ $=\operatorname{Im} \Delta_{*}\left(P_{2}\right)$. Par conséquent, si $\operatorname{Im} \Delta_{*}\left(Q_{2}\right)=\operatorname{Im} \Delta_{*}\left(P_{2}\right)$, alors $\operatorname{Im} \Delta_{* F_{2}}=\operatorname{Im} \Delta_{*}\left(Q_{2}\right)$. Si $\theta_{i}, i=1,2$, est $G_{i}$-équivariante, $q_{2}$ est remplacé par $\left[\left(q_{2}+1\right) / 2\right]$.

En résumé : 
COROLlaIRE 2.9. Pour un sous-feuilletage localement homogène $\left(F_{1}, F_{2}\right)=$ $\left(F_{G_{1}}, F_{G_{2}}\right)$ sur $M=\Gamma \backslash \bar{G} / H$, il y a un diagramme commutatif

$$
\begin{aligned}
& \operatorname{Im} \Delta_{*}\left(Q_{1}\right) \subset \operatorname{Im} \Delta_{*} \supset \operatorname{Im} \Delta_{* F_{2}} \supset \operatorname{Im} \Delta_{*}\left(Q_{2}\right) \\
& \cap \cap \cap \cap \cap \\
& \operatorname{Im} \Delta_{*}\left(P_{1}\right)=\operatorname{Im} \Delta_{*}(P) \supset \operatorname{Im} \Delta_{* F_{2}}(P)=\operatorname{Im} \Delta_{*}\left(P_{2}\right)
\end{aligned}
$$

de sous-algèbres de $H_{D R}(M)$.

Soient $\left(F_{1}, F_{2}\right)=\left(F_{G_{1}}, F_{G_{2}}\right)$ et $\left(F_{1}^{\prime}, F_{2}^{\prime}\right)=\left(F_{G_{1}^{\prime}}, F_{G_{2}^{\prime}}\right)$ deux sous-feuilletages localement homogènes de codimension $\left(q_{1}, q_{2}\right)$ sur $M=\Gamma \backslash \bar{G} / H$ et $M^{\prime}=\Gamma^{\prime} \backslash \bar{G}^{\prime} / H^{\prime}$ respectivement. Supposons qu'il existe un homomorphisme de groupes de Lie $f: \bar{G}^{\prime} \rightarrow \bar{G}$ tel que $f\left(G_{i}^{\prime}\right) \subset G_{i}, i=1,2, f\left(H^{\prime}\right) \subset H$ et $f\left(\Gamma^{\prime}\right) \subset \Gamma$, et tel que les applications linéaires induites $\bar{g}^{\prime} / g_{i}^{\prime} \stackrel{\cong}{\longrightarrow} \bar{g} / g_{i}, i=1,2$, soient des isomorphismes. Alors l'application $\bar{f}: M^{\prime} \rightarrow M$ induite par $f$ est transverse à $\left(F_{1}, F_{2}\right)$. De plus, on a donc $\left(F_{1}^{\prime}, F_{2}^{\prime}\right)=\bar{f}^{-1}\left(F_{1}, F_{2}\right)=\left(\bar{f}^{-1}\left(F_{1}\right), \bar{f}^{-1}\left(F_{2}\right)\right)$. Par suite,

Proposition 2.10. L'homomorphisme caractéristique $\Delta_{*}^{\prime}$ de $\left(F_{1}^{\prime}, F_{2}^{\prime}\right)$ se factorise de la manière suivante:

$$
\Delta_{*}^{\prime}: H\left(W O_{I}\right) \stackrel{\Delta_{*}}{\longrightarrow} H_{D R}(M) \stackrel{\bar{f}^{*}}{\longrightarrow} H_{D R}\left(M^{\prime}\right),
$$

où $\Delta_{*}$ est l'homomorphisme caractéristique de $\left(F_{1}, F_{2}\right)$.

\section{$\S 3$. Fibrés plats.}

Le but de ce paragraphe est d'étudier des cas où l'homomorphisme canonique $\gamma_{*}: H(\bar{g}, H) \rightarrow H_{D R}(M)$ est injectif.

Avec les notations du paragraphe précédent, il est clair que $\gamma *$ est simplement l'homomorphisme caractéristique généralisé $\Delta_{*}(\bar{P})$ du $\bar{G}$-fibré plat $\bar{P}=\Gamma \backslash \bar{G} \times{ }_{H} \bar{G} \rightarrow M=\Gamma \backslash \bar{G} / H$. Si le groupe de Lie $\bar{G}$ est compact, il est évident que l'homomorphisme canonique $\gamma_{*}$ est injectif et que $\Gamma \subset \bar{G}$ est un sous-groupe fini. De même, si $\bar{G}$ est compact connexe, $\gamma_{*}$ est un isomorphisme. Dans le cas où $\bar{G}$ n'est pas nécessairement compact, on démontre sans difficulté le

LEMME 3.1. Soit $H$ un sous-groupe compact d'un groupe de Lie $\bar{G}$. Alors tout sous-groupe discret $\Gamma \subset \bar{G}$ opère de manière proprement discontinue sur $\bar{G} / H$. De plus, si $H=\{e\}$ ou si le sous-groupe discret $\Gamma \subset \bar{G}$ est sans torsion, $\Gamma$ opère sans points fixes sur $\bar{G} / H$; par suite, $M=\Gamma \backslash \bar{G} / H$ est une variété différentiable.

Il est immédiat de vérifier que l'algèbre de Lie $\bar{g}$ d'un groupe de Lie $\bar{G}$ est unimodulaire, pourvu que $\bar{G}$ admette un sous-groupe discret et uniforme $\Gamma$. Nous obtenons alors le résultat suivant.

THÉORÈmE 3.2. Soit $H$ un sous-groupe compact d'un groupe de Lie $\bar{G}$. Supposons que $\bar{G}$ admette un sous-groupe discret et uniforme $\Gamma$. Si $H=\{e\}$ ou si 
$\Gamma$ est sans torsion, alors $M=\Gamma \backslash \bar{G} / H$ est une variété compacte et l'homomorphisme canonique $\gamma_{*}: H(\bar{g}, H) \rightarrow H_{D R}(M)$ est injectif.

Démonstration. La première assertion résulte donc immédiatement du Lemme 3.1. Pour démontrer la seconde assertion, considérons la composante connexe $H_{0}$ (resp. $\bar{G}_{0}$ ) de $H$ (resp. de $\bar{G}$ ) et le sous-groupe discret et uniforme $\Gamma^{\prime}=\Gamma \cap \bar{G}_{0} \subset \bar{G}_{0}$. Soit $\bar{i}: M^{\prime}=\Gamma^{\prime} \backslash \bar{G}_{0} / H_{0} \rightarrow M=\Gamma \backslash \bar{G} / H$ l'application induite par l'inclusion canonique $i: \bar{G}_{0} \rightarrow \bar{G}$. Il est clair que l'homomorphisme canonique $i^{*}: H(\bar{g}, H) \rightarrow H\left(\bar{g}, H_{0}\right)$ est injectif. D'après le Lemme 4.88 dans [9], l'homomorphisme canonique $\gamma_{*}^{\prime}: H\left(\bar{g}, H_{0}\right) \rightarrow H_{D R}\left(M^{\prime}\right)$ est aussi injectif. Puisque $i^{*} \circ \gamma_{*}$ $=\gamma_{*}^{\prime} i^{*}$, il s'ensuit que l'homomorphisme $\gamma_{*}$ est injectif. Ceci achève la démonstration du théorème.

Exemple. Soit $\bar{G}$ l'un des groupes de Lie suivants: $S L(q), G L^{+}(q)$ et $G L(q)$, avec $q \geqq 1$. Soit $H \subset \bar{G}$ un sous-groupe compact. On peut considérer (cf. Borel [1]) un sous-groupe discret, uniforme et sans torsion $\Gamma \subset S L(q)$. Alors, en utilisant l'isomorphisme de groupes de Lie

$$
\phi=(\log , i d) \cdot \phi: G L^{+}(q) \stackrel{\cong}{\longrightarrow} G L^{+}(1) \times S L(q) \stackrel{\cong}{\longrightarrow} R \times S L(q),
$$

avec $\left.\phi(A)=(\operatorname{dét} A \text {, (dét } A)^{-1 / q} \cdot A\right)$ pour $A \in G L^{+}(q)$, on obtient un sous-groupe discret, uniforme et sans torsion $\Gamma_{1}=\phi^{-1}(Z \times \Gamma)$ de $G L^{+}(q)$ et $G L(q)$, où $Z \subset R$ est le groupe additif des entiers. Par suite, les hypothèses du Théorème 3.2 sont vérifiées dans les trois cas précédents.

\section{§4. Sur le calcul de l'homomorphisme $\Delta(\theta)_{*}$.}

Dans ce paragraphe, en utilisant les notations de [11], nous donnons le calcul de l'homomorphisme $\Delta(\theta)_{*}: H\left(W\left(g_{1} \oplus g_{2}, H \times H\right)_{I}\right) \rightarrow H(\bar{g}, H)$. Pour les paires de groupes de Lie $(G, H)$ considérés, on suppose que $I(G) \cong I(g)$, que $H$ ait un nombre fini de composantes connexes et qu'il existe une scission $H$-équivariante $\theta_{0}: g \rightarrow h$ de la suite exacte de $H$-modules $0 \rightarrow h \rightarrow g \rightarrow g / h \rightarrow 0$, où $(g, h)$ est la paire d'algèbres de Lie correspondante.

Soient $\left(G_{j}, H_{j}\right), j=1,2$, deux paires de groupes de Lie, $\left(g_{j}, h_{j}\right)$ leurs paires d'algèbres de Lie. On désigne par $(G, H)$ la paire de groupes de Lie $\left(G_{1} \times G_{2}, H_{1} \times H_{2}\right)$, par $(g, h)$ sa paire d'algèbres de Lie $\left(g_{1} \oplus g_{2}, h_{1} \oplus h_{2}\right)$, et par $i_{0}^{j *}: I\left(G_{j}\right) \rightarrow I\left(H_{j}\right), j=1,2$, les applications de restrictions. Étant donnée une paire $\left(q_{1}, q_{2}\right)$ d'entiers, avec $0 \leqq q_{1} \leqq q_{2}$, considérons des polynômes homogènes invariants $\chi_{\alpha} \in \operatorname{Ker} i_{0}^{1 *} \subset I^{+}\left(G_{1}\right), \alpha=1, \cdots, s$, et $\chi_{\alpha^{\prime}}^{\prime} \in \operatorname{Ker} i_{0}^{2 *} \subset I^{+}\left(G_{2}\right), \alpha^{\prime}=1, \cdots, s^{\prime}$, tels

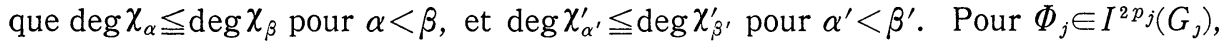
$j=1,2$, avec $0 \leqq p_{1} \leqq q_{1}$ et $0 \leqq p=p_{1}+p_{2} \leqq q_{2}$, posons

$$
z=T_{1} \chi_{1} \cdots T_{s} \chi_{s} \cdot \Phi_{1} \otimes T_{1}^{\prime} \chi_{1}^{\prime} \cdots T_{s^{\prime}}^{\prime} \chi_{s^{\prime}}^{\prime} \cdot \Phi_{2} \in W(g, H) \cong W\left(g_{1}, H_{1}\right) \otimes W\left(g_{2}, H_{2}\right),
$$

où les $T_{\alpha} \chi_{\alpha} \in W^{\text {impair }}\left(g_{1}, H_{1}\right)$ et $T_{\alpha^{\prime}}^{\prime} \chi_{\alpha^{\prime}}^{\prime} \in W^{\text {imgair }}\left(g_{2}, H_{2}\right)$ sont respectivement des 
cochaînes transgressives relatives pour $\chi_{\alpha}$ et $\chi_{\alpha^{\prime}}^{\prime}$ (ceci signifie que $d T_{\alpha} \chi_{\alpha}=\chi_{\alpha}$ et $d T_{\alpha^{\prime}}^{\prime} \chi_{\alpha^{\prime}}^{\prime}=\chi_{\alpha^{\prime}}^{\prime}$ ). Supposons maintenant que les conditions (de cocycle) suivantes soient vérifiées :

(i) $\operatorname{deg} \chi_{1}+2 p_{1} \geqq 2\left(q_{1}+1\right)$ ou $\operatorname{deg} \chi_{1}+2 p \geqq 2\left(q_{2}+1\right)$ pour $s>0$ et $s^{\prime}=0$;

(ii) $\operatorname{deg} \chi_{1}^{\prime}+2 p \geqq 2\left(q_{2}+1\right)$ pour $s=0$ et $s^{\prime}>0$;

(iii) les conditions (i) et (ii) pour $s>0$ et $s^{\prime}>0$.

Alors, par un raisonnement analogue à celui qui a conduit au Lemme 4.33 dans [11], on obtient le résultat suivant.

LEMME 4.1. La cochaine $z \in W(g, H)$ est un cocycle dans $W(g, H)_{I}$ et sa classe de cohomologie $[z] \in H\left(W(g, H)_{I}\right)$ ne dépend pas du choix des cochaînes transgressives relatives $T_{\alpha} \chi_{\alpha}$ et $T_{\alpha^{\prime}}^{\prime} \chi_{\alpha^{\prime}}^{\prime}$ pour $\chi_{\alpha}$ et $\chi_{\alpha^{\prime}}^{\prime}$ respectivement.

Remarques. 1) La classe de cohomologie $[z]$ appartient à l'image de l'homomorphisme canonique $H\left(W\left(g_{1}, H_{1}\right)_{q_{1}}\right) \rightarrow H\left(W(g, H)_{I}\right)$ (resp. $H\left(W\left(g_{2}, H_{2}\right)_{q_{2}}\right)$ $\left.\rightarrow H\left(W(g, H)_{I}\right)\right)$ si $s^{\prime}=p_{2}=0$ (resp. si $\left.s=p_{1}=0\right)$. De même, [z] appartient à l'image de l'homomorphisme canonique $H\left(W(g, H)_{q_{2}}\right) \rightarrow H\left(W(g, H)_{I}\right)$ si $\operatorname{deg} \chi_{1}$ $+2 p \geqq 2\left(q_{2}+1\right)$ pour $s>0$.

2) Le lemme précédent est intéressant dans le cas où, pour chaque $j=1,2$, $\left(g_{j}, h_{j}\right)$ est une $C S$-paire (i. e. une paire réductive d'algèbres de Lie admettant une transgression $\tau_{j}$ pour $g_{j}$ telle que $\operatorname{Ker} i_{0}^{j *}=\operatorname{Idéal}\left(\tau_{j} \hat{P}_{j}\right) \subset I\left(g_{j}\right)$, où $\hat{P}_{j}$ désigne l'espace de Samelson de la paire $\left(g_{j}, h_{j}\right)$ ), parce que (cf. [3] et [9]) il existe un isomorphisme (d'algèbres graduées) $\xi_{*}: H\left(\hat{A}_{I}\right) \otimes_{I(G)} I(H) \stackrel{\cong}{\longrightarrow} H\left(W(g, H)_{I}\right)$ induit par le $D G$-homomorphisme $\xi: \hat{A}_{I} \otimes I(H)=\Lambda \hat{P} \otimes I(G)_{I} \otimes I(H) \cong \Lambda \hat{P}_{1} \otimes \Lambda \hat{P}_{2} \otimes\left(I\left(G_{1}\right) \otimes\right.$ $\left.I\left(G_{2}\right)\right)_{I} \otimes I\left(H_{1}\right) \otimes I\left(H_{2}\right) \rightarrow W(g, H)_{I}$ défini, pour $j=1,2$, par $\xi\left(\Phi_{j}\right)=\Phi_{j}$ pour $\Phi_{j} \in I\left(G_{j}\right)$, $\xi\left(\Psi_{j}\right)=h\left(\theta_{0}^{j}\right)\left(\Psi_{j}\right)$ pour $\Psi_{j} \in I\left(H_{j}\right)$, et $\xi\left(y_{j}\right)=T_{j}\left(\tau_{j} y_{j}\right)$ pour $y_{j} \in \hat{P}_{j}$, où $h\left(\theta_{0}^{j}\right): I\left(H_{j}\right)$ $\rightarrow W\left(g_{j}, H_{j}\right)$ est l'homomorphisme de Chern-Weil d'une scission $H_{j}$-équivariante $\theta_{0}^{j}: g_{j} \rightarrow h_{j}$ de $0 \rightarrow h_{j} \rightarrow g_{j} \rightarrow g_{j} / h_{j} \rightarrow 0$, où $\tau_{j}$ est une transgression pour $g_{j}$ satisfaisant $\operatorname{Ker} i_{0}^{j *}=\operatorname{Idéal}\left(\tau_{j} \hat{P}_{j}\right) \subset I\left(G_{j}\right)$, et où $T_{j}$ est l'opérateur de transgression relatif universel $-\lambda^{1}\left(\theta_{0}^{j}\right): \operatorname{Ker} i_{0}^{j *} \rightarrow W\left(g_{\jmath}, H_{j}\right)$ (voir [11] pour plus de détails).

D'autre part, le calcul de l'homomorphisme $\Delta(\theta)_{*}$ est abordé comme suit. Soient $H \subset G_{2} \subset G_{1} \subset \bar{G}$ des groupes de Lie, $h \subset g_{2} \subset g_{1} \subset \bar{g}$ leurs algèbres de Lie (pas nécessairement réductives). Supposons que, pour chaque $j=1,2$, il existe une scission $H$-équivariante $\theta_{j}: \bar{g} \rightarrow g_{j}$ de la suite exacte de $G_{j}$-modules $0 \rightarrow g_{j} \rightarrow \bar{g} \rightarrow m_{j}=\bar{g} / g_{j} \rightarrow 0$. Pour la paire $\left(q_{1}, q_{2}\right)=\left(\operatorname{dim} \bar{g} / g_{1}, \operatorname{dim} \bar{g} / g_{2}\right)$, considérons l'homomorphisme $\Delta(\theta)_{*}: H\left(W\left(g_{1} \oplus g_{2}, H \times H\right)_{I}\right) \rightarrow H(\bar{g}, H)$ induit par le $H-D G$ homomorphisme $\quad \Delta(\theta)=\mu \circ\left(\Delta\left(\theta_{1}\right) \otimes \Delta\left(\theta_{2}\right)\right): W\left(g_{1} \oplus g_{2}\right) \cong W\left(g_{1}\right) \otimes W\left(g_{2}\right) \rightarrow \Lambda \bar{g}^{*}$ déterminé par l'application linéaire $\theta=\theta_{1}+\theta_{2}: \bar{g} \rightarrow g_{1} \oplus g_{2}$, où $\mu$ désigne la multiplication de $\Lambda \bar{g}^{*}$. Soit maintenant $[z] \in H\left(W\left(g_{1} \oplus g_{2}, H \times H\right)_{I}\right)$ la classe de cohomologie construite dans le Lemme 4.1. Alors, par une technique similaire à celle de Kamber-Tondeur ([11], Proposition 4.58), nous obtenons le résultat suivant.

Proposition 4.2. $\Delta(\theta)_{*}[z]=0 \in H(\bar{g}, H)$ si l'une des conditions suivantes 
est vérifiée: (i) $\Phi_{1} \in I^{+}(\bar{G}) \cdot I\left(G_{1}\right)$; (ii) $\Phi_{2} \in I^{+}(\bar{G}) \cdot I\left(G_{2}\right)$. Si $\theta_{j}, \quad j=1,2$, est $G_{j}$-équivariante, l'idéal $I$ est remplacé par $I^{\prime \prime}$.

Pour le calcul de l'image de $\Delta(\theta)_{*}$, supposons que $(\bar{g}, h)$ soit une $C S$-paire. Considérons les applications de restrictions $i_{j}^{*}: I(\bar{G}) \rightarrow I\left(G_{j}\right), j=1$, et res $: I(\bar{G}) \rightarrow$ $I(H)$. On a alors Ker res $=\operatorname{Idéal}\left\{\bar{c}_{1}, \cdots, \bar{c}_{t}\right\} \subset I(\bar{G}) \cong R\left[\bar{c}_{1}, \cdots, \bar{c}_{r}\right], 0 \leqq t \leqq r=\operatorname{rang} \bar{g}$, avec $\operatorname{deg} \bar{c}_{\alpha} \leqq \operatorname{deg} \bar{c}_{\beta}$ pour $1 \leqq \alpha<\beta \leqq t$. Il s'ensuit que les suspensions relatives $\bar{y}_{\alpha}=\bar{\sigma}_{*} \bar{c}_{\alpha} \in H^{\text {impair }}(\bar{g}, H), \alpha=1, \cdots, t$, des $\bar{c}_{\alpha}$ fournissent une base de l'espace de Samelson de la paire $(\bar{g}, h)$. Par suite, on a

$$
H(\bar{g}, H) \cong \Lambda\left(\bar{y}_{1}, \cdots, \bar{y}_{t}\right) \otimes I(H) / I^{+}(\bar{G}) \cdot I(H) .
$$

D'autre part, notons $2 n_{\alpha}$ le degré de $\bar{c}_{\alpha}$. Supposons qu'il existe deux générateurs $\bar{c}_{j}$ et $\bar{c}_{j^{\prime}}$, avec $1 \leqq j, j^{\prime} \leqq t$ et $j \neq j^{\prime}$, tels que

$$
\begin{aligned}
& i_{1}^{*} \bar{c}_{j}=\sum_{k} \Psi_{j k} . \Phi_{j k} \in I\left(G_{1}\right), \text { avec } \Psi_{j k} \in \operatorname{Ker} i_{0}^{1 *} \text { et } \Phi_{j k} \in I^{2 p_{1}}\left(G_{1}\right), \\
& i_{2}^{*} \bar{c}_{j^{\prime}}=\sum_{k^{\prime}} \Psi_{j^{\prime} k^{\prime}}^{\prime} \cdot \Phi_{j^{\prime} k^{\prime}}^{\prime} \in I\left(G_{2}\right), \text { avec } \Psi_{j^{\prime} k^{\prime}}^{\prime} \in \operatorname{Ker} i_{0}^{2 *} \text { et } \Phi_{j^{\prime} k^{\prime}}^{\prime} \in I^{2 p_{2}}\left(G_{2}\right),
\end{aligned}
$$

et tels que $n_{j} \geqq q_{1}+1$ ou $n_{j}+p_{2} \geqq q_{2}+1$, et $n_{j^{\prime}}+p_{1} \geqq q_{2}+1$. Étant donné un opérateur de transgression relatif universel $T_{1}$ (resp. $T_{2}$ ) pour la paire $\left(G_{1}, H\right)$ (resp. pour la paire $\left(G_{2}, H\right)$ ), démontrons alors le théorème suivant.

ThÉORÈme 4.3. Pour toute suite $1 \leqq i_{1}<\cdots<i_{s} \leqq t$ d'entiers $i_{\alpha} \neq j$, $j^{\prime}$ tels que $n_{i_{1}}+p_{1} \geqq q_{1}+1$ ou $n_{i_{1}}+p_{1}+p_{2} \geqq q_{2}+1$, on a la formule

$$
\begin{aligned}
& \Delta(\theta)_{*_{k, k^{\prime}}}\left[T_{1} i_{1}^{*} \bar{c}_{i_{1}} \cdots T_{1} i_{1}^{*} \bar{c}_{i_{s}} \cdot T_{1} \Psi_{j k} \cdot \Phi_{j k} \otimes T_{2} \Psi_{j^{\prime} k^{\prime}}^{\prime} \cdot \Phi_{\jmath^{\prime} k^{\prime}}^{\prime}\right] \\
& =\bar{y}_{i_{1}} \wedge \cdots \wedge \bar{y}_{i_{s}} \wedge \bar{y}_{j} \wedge \bar{y}_{j^{\prime}} \neq 0 \in H(\bar{g}, H) .
\end{aligned}
$$

Si, de plus, $\theta_{i}, i=1,2$, est $G_{i}$-équivariante, la paire $\left(q_{1}, q_{2}\right)$ est remplacée par la paire $\left(\left[\left(q_{1}+1\right) / 2\right],\left[\left(q_{2}+1\right) / 2\right]\right)$.

Démonstration. Considérons le cas général, puisque la seconde assertion se démontre de façon analogue. Comme

$$
\begin{gathered}
T_{1}^{\prime} i_{1}^{*} \bar{c}_{j}=\sum_{k} T_{1} \Psi_{j k} \cdot \Phi_{j k} \in W\left(g_{1}, H\right) \\
\text { (resp. } \left.T_{2}^{\prime} i_{2}^{*} \bar{c}_{j^{\prime}}=\sum_{k^{\prime}} T_{2} \Psi_{j^{\prime} k^{\prime}}^{\prime} . \Phi_{j^{\prime} k^{\prime}}^{\prime} \in W\left(g_{2}, H\right)\right)
\end{gathered}
$$

est une cochaîne transgressive relative pour $i_{1}^{*} \bar{c}_{j} \in \operatorname{Ker} i_{0}^{1 *}$ (resp. pour $i_{2}^{*} \bar{c}_{j^{\prime}} \in \operatorname{Ker} i_{0}^{2 *}$ ), et comme $n_{j} \geqq q_{1}+1$ ou $n_{j}+p_{2} \geqq q_{2}+1$, et $n_{j^{\prime}}+p_{1} \geqq q_{2}+1$, en utilisant les notations $\mathrm{du}$ Théorème 4.47 dans [11], on obtient alors, pour $n_{i_{1}}+p_{1} \geqq q_{1}+1$ ou $n_{i_{1}}+p_{1}+$ $p_{2} \geqq q_{2}+1$, 


$$
\begin{aligned}
& \bar{y}_{i_{1}} \wedge \cdots \wedge \bar{y}_{i_{s}} \wedge \bar{y}_{j} \wedge \bar{y}_{j^{\prime}} \\
& =\bar{\sigma}_{*} \bar{c}_{i_{1}} \wedge \cdots \wedge \bar{\sigma}_{*} \bar{c}_{i_{s}} \wedge \bar{\sigma}_{*} \bar{c}_{j} \wedge \bar{\sigma}_{*} \bar{c}_{j^{\prime}} \\
& =\left[\bigwedge_{\alpha=1}^{8}\left(\Delta\left(\theta_{1}\right) T_{1} i_{1}^{*} \bar{c}_{i_{\alpha}}-\bar{\pi} \lambda^{1}\left(\theta_{1}\right) \bar{c}_{i_{\alpha}}\right)\right. \\
& \left.\quad \wedge\left(\sum_{k, k^{\prime}} \Delta\left(\theta_{1}\right) T_{1} \Psi_{j k} \cdot \Delta\left(\theta_{1}\right) \Phi_{j k} \cdot \Delta\left(\theta_{2}\right) T_{2} \Psi_{j^{\prime} k^{\prime}}^{\prime} \cdot \Delta\left(\theta_{2}\right) \Phi_{j^{\prime} k^{\prime}}^{\prime}\right)\right] \\
& =\Delta(\theta)_{*} \sum_{k, k^{\prime}}\left[T_{1} i_{1}^{*} \bar{c}_{i_{1}} \cdots T_{1} i_{1}^{*} \bar{c}_{i_{s}} \cdot T_{1} \Psi_{j k} \cdot \Phi_{j k} \otimes T_{2} \Psi_{j^{\prime} k^{\prime}}^{\prime} \cdot \Phi_{j^{\prime} k^{\prime}}^{\prime}\right] \\
& \neq 0 \in H(\bar{g}, H),
\end{aligned}
$$

d'où le théorème.

On en déduit le

CoRollaire 4.4. $\quad \bar{y}_{j} \wedge \bar{y}_{j^{\prime}} \in \operatorname{Im} \Delta(\theta)_{*}^{+}$. Par suite, $\operatorname{dim} \operatorname{Im} \Delta(\theta)_{*}^{+} \geqq 1$. Si, de plus, $p_{1}+p_{2}=q_{2}$, alors

d'où

$$
\operatorname{Idéal}\left(\bar{y}_{j} \wedge \bar{y}_{j^{\prime}}\right)=\left(\bar{y}_{j} \wedge \bar{y}_{j^{\prime}}\right) \cdot \Lambda\left(\bar{y}_{1}, \cdots, \bar{y}_{t}\right) \subset \operatorname{Im} \Delta(\theta)_{*}^{+},
$$

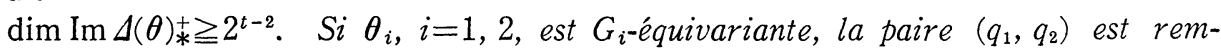
placée par $\left(\left[\left(q_{1}+1\right) / 2\right],\left[\left(q_{2}+1\right) / 2\right]\right)$.

Remarque. Dans le cas où $p_{1}=q_{1}$, en vertu du Théorème 4.47 dans [11], on a donc

$$
\begin{aligned}
& \Delta(\theta)_{*} \sum_{k}\left[T_{1} i_{1}^{*} \bar{c}_{i_{1}} \cdots T_{1} i_{1}^{*} \bar{c}_{i_{s}} \cdot T_{1} \Psi_{j k} \cdot \Phi_{j k} \otimes 1\right] \\
& =\bar{y}_{i_{1}} \wedge \cdots \wedge \bar{y}_{i_{s}} \wedge \bar{y}_{\jmath} \neq 0 \in H(\bar{g}, H)
\end{aligned}
$$

pour $1 \leqq i_{1}<\cdots<i_{s} \leqq t, 0 \leqq s \leqq t-1$, avec $i_{\alpha} \neq j$. Par conséquent,

$$
\operatorname{Idéal}\left(\bar{y}_{j}\right)=\bar{y}_{j} \cdot \Lambda\left(\bar{y}_{1}, \cdots, \bar{y}_{t}\right) \subset \operatorname{Im} \Delta\left(\theta_{1}\right)_{*}^{+} \subset \operatorname{Im} \Delta(\theta)_{*}^{+},
$$

d'où $\operatorname{dim} \operatorname{Im} \Delta(\theta)_{*}^{+} \geqq 2^{t-1}$. De même, pour toute suite $t^{\prime}<i_{1}<\cdots<i_{s} \leqq t$ d'entiers $i_{\alpha}$, on obtient $\Delta(\theta)_{*}\left[T_{1} i_{1}^{*} \bar{c}_{i_{1}} \cdots T_{1} i_{1}^{*} \bar{c}_{i_{s}} \otimes 1\right]=\bar{y}_{i_{1}} \wedge \cdots \wedge \bar{y}_{i_{s}} \neq 0 \in H(\bar{g}, H)$, où $t^{\prime}$ est le plus petit entier, $1 \leqq t^{\prime} \leqq t$, tel que $n_{\alpha}>q_{1}$ pour $t^{\prime}<\alpha \leqq t$. Par suite, on a alors $\Lambda\left(\bar{y}_{t^{\prime}+1}, \cdots, \bar{y}_{t}\right) \subset \operatorname{Im} \Delta\left(\theta_{1}\right)_{*} \subset \operatorname{Im} \Delta(\theta)_{*}$, d'où $\operatorname{dim} \operatorname{Im} \Delta(\theta)_{*} \geqq 2^{t-t^{\prime}}$. Si $\theta_{1}$ est $G_{1}$-équivariante, on peut remplacer $q_{1}$ par $\left[\left(q_{1}+1\right) / 2\right]$.

\section{§5. Calcul de l'homomorphisme $W(d \rho)^{*}$.}

Dans ce paragraphe on réduit le calcul de l'homomorphisme $W(d \rho)^{*}$ : $H\left(W\left(g^{\prime}, H^{\prime}\right)_{I}\right) \rightarrow H\left(W(g, H)_{I}\right)$ à celui d'un homomorphisme (d'algèbres graduées) $\hat{\rho}^{*}: H\left(\hat{A}_{I}^{\prime}\right) \rightarrow H\left(\hat{A}_{I}\right)$. Pour cela, nous supposons que les paires d'algèbres de Lie $(g, h)$ des paires de groupes de Lie considérés $(G, H)$ soient $C S$-paires, que $I(G) \cong I(g)$ et que $H$ ait un nombre fini de composantes connexes.

Soient $(G, H)$ et $\left(G^{\prime}, H^{\prime}\right)$ des paires de groupes de Lie (vérifiant les hypothèses précédentes), $(g, h)$ et $\left(g^{\prime}, h^{\prime}\right)$ leurs paires d'algèbres de Lie. Considérons 
les applications de restrictions $i_{0}^{*}: I(G) \rightarrow I(H)$ et $i_{0}^{\prime *}: I\left(G^{\prime}\right) \rightarrow I\left(H^{\prime}\right)$. On a alors Ker $i_{0}^{*}=\operatorname{Idé} a 1\left\{c_{1}, \cdots, c_{t}\right\} \subset I(G) \cong R\left[c_{1}, \cdots, c_{r}\right], \quad 0 \leqq t \leqq r=\operatorname{rang} g, \quad$ avec $\operatorname{deg} c_{\alpha} \leqq$ $\operatorname{deg} c_{\beta}$ pour $1 \leqq \alpha<\beta \leqq t$. De même, on obtient donc $\operatorname{Ker} i_{0}^{\prime *}=\operatorname{Idéal}\left\{c_{1}^{\prime}, \cdots, c_{t^{\prime}}^{\prime}\right\} \subset$ $I\left(G^{\prime}\right) \cong R\left[c_{1}^{\prime}, \cdots, c_{r^{\prime}}^{\prime}\right], 0 \leqq t^{\prime} \leqq r^{\prime}=\operatorname{rang} g^{\prime}$, avec $\operatorname{deg} c_{\alpha}^{\prime} \leqq \operatorname{deg} c_{\beta}^{\prime}$ pour $1 \leqq \alpha<\beta \leqq t^{\prime}$. Il s'ensuit que les suspensions relatives $y_{\alpha}=\sigma_{*} c_{\alpha} \in H^{\text {impair }}(g, H), 1 \leqq \alpha \leqq t$ (resp. $\left.y_{\alpha}^{\prime}=\sigma_{*}^{\prime} c_{\alpha}^{\prime} \in H^{\text {impair }}\left(g^{\prime}, H^{\prime}\right), 1 \leqq \alpha \leqq t^{\prime}\right)$ des $c_{\alpha} \in \operatorname{Ker} i_{0}^{*}$ (resp. des $c_{\alpha}^{\prime} \in \operatorname{Ker} i_{0}^{\prime *}$ ) fournissent une base de l'espace de Samelson $\hat{P}$ (resp. $\left.\hat{P}^{\prime}\right)$ de la paire $(g, h)$ (resp. de la paire $\left.\left(g^{\prime}, h^{\prime}\right)\right)$.

D'autre part, supposons qu'il existe un homomorphisme de groupes de Lie $\rho: G \rightarrow G^{\prime}$ tel que $\rho(H) \subset H^{\prime}$. On désigne par $\rho_{0}: H \rightarrow H^{\prime}$ l'homomorphisme de groupes de Lie induit par $\rho$. En particulier, l'homomorphisme $\rho^{*}: I\left(G^{\prime}\right) \rightarrow I(G)$ déterminé par $\rho$ applique $\operatorname{Ker} i_{0}^{*}$ dans $\operatorname{Ker} i_{0}^{*}$. Il est immédiat de voir que, pour chaque $\alpha=1, \cdots, t^{\prime}$, l'élément $\rho^{*} c_{\alpha}^{\prime} \in \operatorname{Ker} i_{0}^{*} \subset I^{+}(G)$ peut s'écrire d'une façon et d'une seule sous la forme

$$
\begin{gathered}
\rho^{*} c_{\alpha}^{\prime}=\sum_{\beta=1}^{t} c_{\beta}\left(a_{\alpha \beta}+\Phi_{\alpha \beta}\right), \\
\text { avec } a_{\alpha \beta} \in R \cong I^{0}(G) \text { et } \Phi_{\alpha \beta} \in R\left[c_{\beta}, \cdots, c_{r}\right]^{+} \subset I^{+}(G) .
\end{gathered}
$$

De plus, si $\rho^{*} c_{\alpha}^{\prime} \neq 0$, on obtient $\operatorname{deg} c_{\alpha}^{\prime}=\operatorname{deg} c_{\beta}$ pour $a_{\alpha \beta} \neq 0$, et $\operatorname{deg} c_{\alpha}^{\prime}=\operatorname{deg} c_{\beta}+$ $\operatorname{deg} \Phi_{\alpha \beta}$ pour $\Phi_{\alpha \beta} \neq 0$; par suite, $a_{\alpha \beta} \cdot \Phi_{\alpha \beta}=0$ pour $\alpha=1, \cdots, t^{\prime}$ et $\beta=1, \cdots, t$.

Étant donné un entier $q \geqq 0$, considérons maintenant le $D G$-homomorphisme

$$
\hat{\rho}: \hat{A}_{q}^{\prime}=\Lambda \hat{P}^{\prime} \otimes I\left(G^{\prime}\right)_{q} \rightarrow \hat{A}_{q}=\Lambda \hat{P} \otimes I(G)_{q}
$$

induit par $\rho^{*}$ sur $I\left(G^{\prime}\right)_{q}$ et caractérisé comme homomorphisme d'algèbres graduées sur $\Lambda \hat{P}^{\prime}$ par la formule

$$
\hat{\rho}\left(y_{\alpha}^{\prime}\right)=\sum_{\beta=1}^{t} y_{\beta} \otimes\left(a_{\alpha \beta}+\Phi_{\alpha \beta}\right) \quad \text { pour } \alpha=1, \cdots, t^{\prime} .
$$

Alors le calcul de l'homomorphisme $W(d \rho)^{*}: H\left(W\left(g^{\prime}, H^{\prime}\right)_{q}\right) \rightarrow H\left(W(g, H)_{q}\right)$ se fait à l'aide du théorème suivant.

THEORÈmE 5.1. L'homomorphisme $W(d \rho)^{*}$ se factorise de la manière suivante:

$$
\begin{aligned}
W(d \rho)^{*}: H\left(W\left(g^{\prime}, H^{\prime}\right)_{q}\right) \stackrel{\xi_{*}^{\prime-1}}{\cong} H\left(\hat{A}_{q}^{\prime}\right) \otimes_{I\left(G^{\prime}\right)} I\left(H^{\prime}\right) \stackrel{\hat{\rho}^{*} \otimes \rho_{0}^{*}}{\longrightarrow} \\
H\left(\hat{A}_{q}\right) \otimes_{I(G)} I(H) \stackrel{\xi_{*}}{\cong} H\left(W(g, H)_{q}\right) .
\end{aligned}
$$

Démonstration. Il suffit de prouver que le diagramme suivant est commutatif :

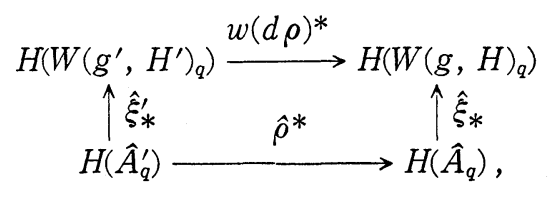


où $\hat{\xi}_{*}$ et $\hat{\xi}_{*}^{\prime}$ désignent respectivement les homomorphismes induits en cohomologie par les $D G$-homomorphismes déterminés par les formules:

$$
\begin{aligned}
& \hat{\xi}(\Phi)=\Phi \text { pour } \Phi \in I(G) \text { et } \hat{\xi}\left(y_{\alpha}\right)=T_{0} c_{\alpha} \text { pour } \alpha=1, \cdots, t, \\
& \hat{\xi}^{\prime}\left(\Phi^{\prime}\right)=\Phi^{\prime} \text { pour } \Phi^{\prime} \in I\left(G^{\prime}\right) \text { et } \hat{\xi}^{\prime}\left(y_{\alpha}^{\prime}\right)=T_{0}^{\prime} c_{\alpha}^{\prime} \text { pour } \alpha=1, \cdots, t^{\prime},
\end{aligned}
$$

$T_{0}$ (resp. $T_{0}^{\prime}$ ) étant l'opérateur de transgression relatif universel pour $(G, H)$ (resp. pour $\left(G^{\prime}, H^{\prime}\right)$ ), déterminé par une scission $H$-équivariante $\theta_{0}: g \rightarrow h$ (resp. $H^{\prime}$-équivariante $\theta_{0}^{\prime}: g^{\prime} \rightarrow h^{\prime}$ ) de $0 \rightarrow h \rightarrow g \rightarrow g / h \rightarrow 0$ (resp. de $0 \rightarrow h^{\prime} \rightarrow g^{\prime} \rightarrow g^{\prime} / h^{\prime} \rightarrow 0$ ).

Pour montrer que $W(d \rho)^{*}{ }_{\circ} \hat{\xi}_{*}^{\prime}=\hat{\xi}_{*}^{\circ} \hat{\rho}^{*}$, considérons la base de Vey de $H\left(\hat{A}_{q}^{\prime}\right)$. fournie (cf. [9], Théorème 5.110) par les classes de cohomologie des cocycles monômiaux

$$
z_{(i, j)}^{\prime}=y_{(i)}^{\prime} \otimes c_{(j)}^{\prime}=y_{i_{1}}^{\prime} \wedge \cdots \wedge y_{i_{s}}^{\prime} \otimes c_{1}^{\prime j_{1}} \cdots c_{r^{\prime}}^{\prime j_{r^{\prime}}}
$$

avec $j_{\alpha} \geqq 0,0 \leqq \operatorname{deg} c_{(j)}^{\prime} \leqq 2 q, 1 \leqq i_{1}<\cdots<i_{s} \leqq t^{\prime}, \quad 0 \leqq s \leqq t^{\prime}, \quad \operatorname{deg} c_{i_{1}}^{\prime}+\operatorname{deg} c_{(j)}^{\prime} \geqq 2(q+1)$ pour $s>0, j_{\alpha}=0$ pour $\alpha<i_{1}$ si $s>0$, et $j_{\alpha}=0$ pour $\alpha=1, \cdots, t^{\prime}$ si $s=0$. Puisque, pour chaque $\alpha=1, \cdots, s$,

$$
W(d \rho) T_{0}^{\prime} c_{i_{\alpha}}^{\prime}, \sum_{\beta=1}^{t} T_{0} c_{\beta} \cdot\left(a_{i_{\alpha \beta}}+\Phi_{i_{\alpha \beta}}\right) \in W^{\text {impair }}(g, H)
$$

sont deux cochaînes transgressives relatives pour $\rho^{*} c_{i_{\alpha}}^{\prime} \in \operatorname{Ker} i_{v}^{*}$, on obtient donc, en vertu du Lemme 4.1,

$$
\begin{aligned}
W(d \rho)^{*}\left(\hat{\xi}_{*}^{\prime}\left[z_{(i, j)}^{\prime}\right]\right) & =\left[W(d \rho) T_{0}^{\prime} c_{i_{1}}^{\prime} \cdots W(d \rho) T_{0}^{\prime} c_{i_{s}}^{\prime} \cdot \rho^{*} c_{(j)}^{\prime}\right] \\
& =\left[\left(\sum_{\beta=1}^{t} T_{0} c_{\beta} \cdot\left(a_{i_{1 \beta}}+\Phi_{i_{1 \beta}}\right)\right) \cdots\left(\sum_{\beta=1}^{t} T_{0} c_{\beta} \cdot\left(a_{i_{s \beta}}+\Phi_{i_{s \beta}}\right)\right) \cdot \rho^{*} c_{(j)}^{\prime}\right] \\
& =\hat{\xi}_{*}\left(\hat{\rho}^{*}\left[z_{(i, j)}^{\prime}\right]\right) .
\end{aligned}
$$

Ceci achève la démonstration du théorème.

Remarques. 1) Il est clair que l'homomorphisme $\hat{\rho}^{*}$ est défini sur la base de Vey de $H\left(\hat{A}_{q}^{\prime}\right)$ par la formule

$$
\hat{\rho}^{*}\left[z_{(i, \jmath)}^{\prime}\right]=\left[\left(\sum_{\beta=1}^{t} y_{\beta} \otimes\left(a_{i_{1 \beta}}+\Phi_{i_{1 \beta}}\right)\right) \cdots\left(\sum_{\beta=1}^{t} y_{\beta} \otimes\left(a_{i_{s \beta}}+\Phi_{i_{s \beta}}\right)\right) \cdot \rho^{*} c_{(\jmath)}^{\prime}\right] .
$$

En particulier, pour $\operatorname{deg} c_{(j)}^{\prime}=2 q$, on a alors

$$
\hat{\rho}^{*}\left[z_{(i, j)}^{\prime}\right]=\left[\left(\sum_{\beta=1}^{t} y_{\beta} a_{i_{1 \beta}}\right) \wedge \cdots \wedge\left(\sum_{\beta=1}^{t} y_{\beta} a_{i_{s \beta}}\right) \otimes \rho^{*} c_{(j)}^{\prime}\right] .
$$

2) Comme l'homomorphisme $\hat{\xi}_{*}=\hat{\xi}_{*(G, H)}$ est injectif pour paires de groupes de Lie $(G, H)$, il en résulte que la construction de $\hat{\rho}^{*}$ est fonctoriel pour homomorphismes de paires de groupes de Lie

$$
\rho:(G, H) \rightarrow\left(G^{\prime}, H^{\prime}\right) .
$$


3) $\mathrm{Si}$, de plus, les applications de restrictions $i_{0}^{*}$ et $i_{0}^{* *}$ sont surjectives, l'homomorphisme $W(d \rho)^{*}$ est donné par la composition

$$
W(d \rho)^{*}: H\left(W\left(g^{\prime}, H^{\prime}\right)_{q}\right) \stackrel{\hat{\xi}_{*}^{\prime-1}}{\cong} H\left(\hat{A}_{q}^{\prime}\right) \stackrel{\hat{\rho}^{*}}{\longrightarrow} H\left(\hat{A}_{q}\right) \stackrel{\hat{\xi}_{*}}{\cong} H\left(W(g, H)_{z}\right) .
$$

Soit maintenant $\left[z_{(i, j)}^{\prime}\right]=\left[y_{(i)}^{\prime} \otimes c_{(j)}^{\prime}\right]$ un élément de la base de Vey de $H\left(\hat{A}_{q}^{\prime}\right)$ $\subset H\left(W\left(g^{\prime}, H^{\prime}\right)_{q}\right)$. On en déduit le

Corollaire 5.2. Si $\operatorname{deg} c_{(j)}^{\prime}=2 q$ et $s^{\prime} i l$ existe un $\alpha=1, \cdots, s$ (avec $s>0$ ) tel que $a_{i_{\alpha \beta}}=0$ pour tout $\beta=1, \cdots, t$, alors

$$
\left[z_{(i, j)}^{\prime}\right] \in \operatorname{Ker} W(d \rho)^{*} .
$$

On peut appliquer ce résultat au problème du prolongement de feuilletages comme suit. Étant donnés des entiers $q_{1}, q_{2}$ et $d$, avec $0<q_{1}<q_{2}$ et $d=q_{2}-q_{1}>0$, prenons $(G, H)=\left(G L\left(q_{1}\right) \times G L(d), O\left(q_{1}\right) \times O(d)\right),\left(G^{\prime}, H^{\prime}\right)=\left(G L\left(q_{2}\right), O\left(q_{2}\right)\right)$ et $\rho:(G, H)$ $\rightarrow\left(G^{\prime}, H^{\prime}\right)$ l'inclusion canonique. Il est bien évident que

et que

$$
\begin{gathered}
H\left(W(g, H)_{q_{2}}\right) \cong H\left(\hat{A}_{q_{2}}\right)=H\left(\Lambda\left(y_{1}, y_{3}, \cdots, y_{2 q_{1}^{\prime}-1}\right) \otimes \Lambda\left(y_{1}^{\prime}, y_{3}^{\prime}, \cdots, y_{2 d^{\prime}-1}^{\prime}\right)\right. \\
\left.\otimes\left(R\left[c_{1}, c_{2}, \cdots, c_{q_{1}}\right] \otimes R\left[c_{1}^{\prime}, c_{2}^{\prime}, \cdots, c_{d}^{\prime}\right]\right)_{q_{2}}\right)
\end{gathered}
$$

$$
\begin{aligned}
& H\left(W\left(g^{\prime}, H^{\prime}\right)_{q_{2}}\right) \cong H\left(\hat{A}_{q_{2}}^{\prime}\right)=H\left(W O_{q_{2}}\right) \\
& =H\left(\Lambda\left(y_{1}^{\prime \prime}, y_{3}^{\prime \prime}, \cdots, y_{2 q_{2}-1}^{\prime \prime}\right) \otimes R\left[c_{1}^{\prime \prime}, c_{2}^{\prime \prime}, \cdots, c_{q_{2}}^{\prime \prime}\right]_{q_{2}}\right),
\end{aligned}
$$

où les éléments $y_{i}, y_{i}^{\prime}$ et $y_{i}^{\prime \prime}$ (de degrés $2 i-1$ ) sont respectivement les suspensions relatives des polynômes de Chern $c_{i} \in I\left(G L\left(q_{1}\right)\right) \cong R\left[c_{1}, \cdots, c_{q_{1}}\right], c_{i}^{\prime} \in I(G L(d))$ $\cong R\left[c_{1}^{\prime}, \cdots, c_{d}^{\prime}\right]$ et $c_{i}^{\prime \prime} \in I\left(G L\left(q_{2}\right)\right) \cong R\left[c_{1}^{\prime \prime}, \cdots, c_{q_{2}}^{\prime \prime}\right]$ de degrés $2 i$, avec $i$ impair, et où $q_{i}^{\prime}=\left[\left(q_{i}+1\right) / 2\right], i=1,2$, et $d^{\prime}=[(d+1) / 2]$.

D'autre part, comme l'homomorphisme $\rho^{*}: I\left(G^{\prime}\right) \rightarrow I(G) \cong I\left(G L\left(q_{1}\right)\right) \otimes I(G L(d))$ est donné, pour chaque $j=1, \cdots, q_{2}$, par la formule

$$
\rho^{*} c_{j}^{\prime \prime}=\sum_{i=0}^{j} c_{i} \otimes c_{\jmath-i}^{\prime}, \quad \text { avec } c_{i}=0 \text { pour } i>q_{1}, \quad c_{i}^{\prime}=0 \text { pour } i>d
$$

et $c_{0}=c_{0}^{\prime}=1$, on obtient donc

$$
\hat{\rho}\left(y_{2 j-1}^{\prime \prime}\right)=\sum_{i=1}^{j} y_{2 i-1} \otimes 1 \otimes 1 \otimes c_{2(j-i)}^{\prime}+\sum_{i=1}^{j} 1 \otimes y_{2 i-1}^{\prime} \otimes c_{2(j-i)} \otimes 1
$$

pour $j=1, \cdots, q_{2}^{\prime}$, avec $y_{2 i-1}=0$ pour $i>q_{1}^{\prime}$ et $y_{2 i-1}^{\prime}=0$ pour $i>d^{\prime}$. Considérons la base de Vey de $H\left(W O_{q_{2}}\right)$ fournie par les classes de cohomologie des cocycles monômiaux

$$
z_{(i, j)}=y_{(2 i-1)}^{\prime \prime} \otimes c_{(j)}^{\prime \prime}=y_{2 i_{1}-1}^{\prime \prime} \wedge \cdots \wedge y_{2 i_{s}-1}^{\prime \prime} \otimes c_{1}^{\prime \prime}{ }^{j} \cdots c_{q_{2}}^{\prime \prime j} q_{2}
$$

avec $j_{\alpha} \geqq 0,0 \leqq 2 p=\operatorname{deg} c_{(j)}^{\prime \prime} \leqq 2 q_{2}, 1 \leqq i_{1}<\cdots<i_{s} \leqq q_{2}^{\prime}, 0 \leqq s \leqq q_{2}^{\prime}, i_{1}+p \geqq q_{2}+1$ pour $s>0, \quad j_{\alpha}=0$ pour tout entier impair $\alpha<2 i_{1}-1$ si $s>0$, et $j_{\alpha}=0$ pour tout entier 
impair $\alpha$ si $s=0$. D'après le Corollaire 5.2 , si $p=q_{2}$ et s'il existe un $\alpha=1, \cdots, s$ (avec $s>0$ ) tel que $i_{\alpha} \geqq \max \left(q_{1}^{\prime}, d^{\prime}\right)+1$, alors on a $\left[z_{(i, j)}\right] \in \operatorname{Ker} W(d \rho)^{*}$.

Soit maintenant $\left(F_{1}, F_{2}\right)$ un sous-feuilletage de codimension $\left(q_{1}, q_{2}\right)$ sur une variété différentiable $M$ de dimension $n$. Alors, en vertu du Théorème 6.1 dans [4], on a donc $\operatorname{Ker} W(d \rho)^{*} \subset \operatorname{Ker} \Delta_{*}\left(Q_{2}\right)$, où $\Delta_{*}\left(Q_{2}\right)$ désigne l'homomorphisme caractéristique du feuilletage $F_{2}$. Par suite, on a obtenu le résultat suivant.

THÉORÈme 5.3. Soit $F_{2}$ un feuilletage de codimension $q_{2}$ sur $M$. Si $p=q_{2}$ et $s^{\prime} i l$ existe un $\alpha=1, \cdots, s$ (avec $s>0$ ) vérifiant $i_{\alpha} \geqq \max \left(q_{1}^{\prime}, d^{\prime}\right)+1$ tel $\overrightarrow{q u e}$ $\Delta_{*}\left(Q_{2}\right)\left[z_{(i, j)}\right] \neq 0 \in H_{D R}(M)$, aucun feuilletage intégrablement homotope à $F_{2}$ ne peut se prolonger en un feuilletage $F$ de codimension $q$ sur $M$, avec $q_{1} \leqq q \leqq d$ si $q_{1} \leqq d$, et $d \leqq q \leqq q_{1}$ si $q_{1}>d$.

Pour un feuilletage $F_{2}$ de codimension $q_{2} \geqq 3$ sur $M$, on considère un cocycle de la forme $z=y_{1}^{\prime \prime} \wedge y_{2 q_{2}-1}^{\prime \prime \prime} \otimes c_{(j)}^{\prime \prime}$ avec $\operatorname{deg} c_{(j)}^{\prime \prime}=2 q_{2}$. On en déduit le

Corollaire 5.4. Si la classe $\Delta_{*}\left(Q_{2}\right)[z] \in H_{D R}(M)$ n'est pas nulle, aucun feuilletage intégrablement homotope à $F_{2}$ ne peut se prolonger en un feuilletage $F_{1}$ de codimension $q_{1}$ sur $M$ pour $0<q_{1}<q_{2}$, sauf dans les cas où $\left(q_{1}, q_{2}\right)=\left(2 q_{2}^{\prime}-1,2 q_{2}^{\prime}\right)$ ou $\left(q_{1}, q_{2}\right)=\left(1,2 q_{2}^{\prime}\right)$.

Il est clair que, pour $q_{2}=2 q_{2}^{\prime} \geqq 2$, aucun feuilletage intégrablement homotope à $F_{2}$ ne peut se prolonger en un feuilletage de codimension impaire, pourvu que $Q_{2}=T M / F_{2}$ soit un fibré vectoriel orienté ayant une classe de Pfaff non nulle.

Exemple. Compte tenu du Théorème 5.37 dans [11], on voit donc que, pour $q_{2} \geqq 2$, toutes les hypothèses précédentes sont vérifiées par le feuilletage localement homogène $F_{2}=F_{S L\left(q_{2}+1,1\right)_{0}}$ (resp. $F_{2}=F_{G L+\left(q_{2}+1,1\right)_{0}}$ ) de codimension $q_{2}$ sur $M=\Gamma \backslash S L\left(q_{2}+1\right) / S O\left(q_{2}\right)$ (resp. sur $\left.M=\Gamma^{\wedge} \backslash G L^{+}\left(q_{2}+1\right) / S O\left(q_{2}\right)\right)$, où les groupes de Lie $S L\left(q_{2}+1,1\right)_{0}$ et $G L^{+}\left(q_{2}+1,1\right)_{0}$ seront donnés dans le $\S 6$, et où $\Gamma$ (resp. $\Gamma^{\prime}$ ) est un sous-groupe discret, uniforme et sans torsion de $S L\left(q_{2}+1\right)$ (resp. de $\left.G L^{+}\left(q_{2}+1\right)\right)$.

Remarque. Dans le cas où $\left(F_{1}, F_{2}\right)$ est un sous-feuilletage de codimension $\left(q_{1}, q_{2}\right)$ sur $M$ à fibré normal trivialisé, on obtient donc $\Delta_{*}\left(Q_{2}\right)\left[z_{(i, j)}\right]=0 \in H_{D R}(M)$ si $p=q_{2}$ et s'il existe un $\alpha=1, \cdots, s$ tel que $i_{\alpha} \geqq \max \left(q_{1}, d\right)+1$, où $\left[z_{(i, j)}\right]$ est un élément de la base de Vey de

$$
H^{+}\left(W\left(g l\left(q_{2}\right)\right)_{q_{2}}\right) \cong H^{+}\left(W_{q_{2}}\right)=H^{+}\left(\Lambda\left(y_{1}^{\prime \prime}, y_{2}^{\prime \prime}, \cdots, y_{q_{2}}^{\prime \prime}\right) \otimes R\left[c_{1}^{\prime \prime}, c_{2}^{\prime \prime}, \cdots, c_{q_{2}}^{\prime \prime}\right]_{q_{2}}\right)
$$

fournie par les classes de cohomologie des cocycles monômiaux

$$
z_{(i, j)}=y_{(i)}^{\prime \prime} \otimes c_{(j)}^{\prime \prime}=y_{i_{1}}^{\prime \prime} \wedge \cdots \wedge y_{i_{s}}^{\prime \prime} \otimes c_{1}^{\prime \prime j_{1}} \cdots c_{q_{2}}^{\prime \prime q_{2}}
$$

avec $j_{\alpha} \geqq 0, \quad 0 \leqq 2 p=\operatorname{deg} c_{(j)}^{\prime \prime} \leqq 2 q_{2}, \quad 1 \leqq i_{1}<\cdots<i_{s} \leqq q_{2}, \quad 0<s \leqq q_{2}, \quad i_{1}+p \geqq q_{2}+1$, et $j_{\alpha}=0$ pour $\alpha<i_{1}$. D'autre part, soit maintenant $F_{2}$ un feuilletage de codimen- 
sion $q_{2} \geqq 2$ sur $M$ à fibré normal trivialisé. On considère un cocycle de la forme $z=y_{1}^{\prime \prime} \wedge y_{q_{2}}^{\prime \prime} \otimes c_{(j)}^{\prime \prime}$ avec $\operatorname{deg} c_{(j)}^{\prime \prime}=2 q_{2}$. Si la classe $\Delta_{*}\left(Q_{2}\right)[z] \in H_{D R}(M)$ n'est pas nulle, il est évident qu'un feuilletage $F_{2}^{\prime}$ (à fibré normal trivialisé) intégrablement homotope à $F_{2}$ ne peut pas se prolonger en un feuilletage $F_{1}$ de codimension $q_{1}$ sur $M$ tel que le fibré normal du sous-feuilletage $\left(F_{1}, F_{2}^{\prime}\right)$ de codimension $\left(q_{1}, q_{2}\right)$ soit trivialisé, avec $0<q_{1}<q_{2}$. C'est par exemple le cas si $F_{2}$ est le feuilletage localement homogène $F_{S L\left(q_{2}+1,1\right)_{0}}$ sur $M=\Gamma \backslash S L\left(q_{2}+1\right)$ avec $q_{2} \geqq 2$, où $\Gamma \subset S L\left(q_{2}+1\right)$ est un sous-groupe discret et uniforme. Un autre exemple de ce cas est obtenu en prenant $F_{2}=F_{G L^{+}\left(q_{2}+1,1\right)_{0}}$ et $M=\Gamma \backslash G L^{+}\left(q_{2}+1\right)$ avec $q_{2} \geqq 2$, où $\Gamma \subset G L^{+}\left(q_{2}+1\right)$ est un sous-groupe discret et uniforme.

D'autre part, le calcul de l'homomorphisme $W(d \rho)^{*}: H\left(W\left(g^{\prime}, H^{\prime}\right)_{I}\right) \rightarrow$ $H\left(W(g, H)_{I}\right)$ peut se faire comme suit. Soient $\left(G_{\imath}, H_{i}\right)$ et $\left(G_{i}^{\prime}, H_{i}^{\prime}\right), i=1,2$, des paires de groupes de Lie (vérifiant les hypothèses usuelles de ce paragraphe). Supposons que, pour chaque $i=1,2$, il existe un homomorphisme de paires de groupes de Lie $\rho_{i}:\left(G_{i}, H_{i}\right) \rightarrow\left(G_{i}^{\prime}, H_{i}^{\prime}\right)$. Considérons maintenant l'homomorphisme de paires de groupes de Lie

$$
\rho=\left(\rho_{1}, \rho_{2}\right):(G, H)=\left(G_{1} \times G_{2}, H_{1} \times H_{2}\right) \rightarrow\left(G^{\prime}, H^{\prime}\right)=\left(G_{1}^{\prime} \times G_{2}^{\prime}, H_{1}^{\prime} \times H_{2}^{\prime}\right) .
$$

Étant donnée une paire $\left(q_{1}, q_{2}\right)$ d'entiers, avec $0 \leqq q_{1} \leqq q_{2}$, par une technique identique à celle du Théorème 5.1 , on construit un homomorphisme de $D G$ algèbres $\hat{\rho}: \hat{A}_{I}^{\prime}=\Lambda \hat{P}^{\prime} \otimes I\left(G^{\prime}\right)_{I} \rightarrow \hat{A}_{I}=\Lambda \hat{P} \otimes I(G)_{I}$. Ainsi, en reprenant les notations du Théorème 5.1 , par un raisonnement identique à celui utilisé dans la démonstration de ce théorème, nous obtenons le résultat suivant.

THÉORÈme 5.5. Pour l'homomorphisme $W(d \rho)^{*}$, on a la factorisation suivante:

$$
\begin{aligned}
W(d \rho)^{*}: H\left(W\left(g^{\prime}, H^{\prime}\right)_{I}\right) & \stackrel{\xi_{*}^{\prime-1}}{\longrightarrow} H\left(\hat{A}_{I}^{\prime}\right) \otimes_{I\left(G^{\prime}\right)} I\left(H^{\prime}\right) \stackrel{\hat{\rho}^{*} \otimes \rho_{0}^{*}}{\longrightarrow} H\left(\hat{A}_{I}\right) \otimes_{I(G)} I(H) \\
& \stackrel{\xi_{*}}{\longrightarrow} H\left(W(g, H)_{I}\right) .
\end{aligned}
$$

Remarques. 1) Comme l'homomorphisme $\hat{\xi}_{*}=\hat{\xi}_{*(G, H)}: H\left(\hat{A}_{I}\right) \rightarrow H\left(W(g, H)_{I}\right)$ est injectif pour paires de groupes de Lie $(G, H)=\left(G_{1} \times G_{2}, H_{1} \times H_{2}\right)$, il s'ensuit que la construction de l'homomorphisme $\hat{\rho}^{*}$ est fonctoriel pour homomorphismes de paires de groupes de Lie

$$
\rho=\left(\rho_{1}, \rho_{2}\right):(G, H)=\left(G_{1} \times G_{2}, H_{1} \times H_{2}\right) \rightarrow\left(G^{\prime}, H^{\prime}\right)=\left(G_{1}^{\prime} \times G_{2}^{\prime}, H_{1}^{\prime} \times H_{2}^{\prime}\right) .
$$

2) $\mathrm{Si}$, de plus, les applications de restrictions $i_{0}^{*}$ et $i_{0}^{\prime *}$ sont surjectives, l'homomorphisme $W(d \rho)^{*}$ se factorise de la manière suivante:

$$
W(d \rho)^{*}: H\left(W\left(g^{\prime}, H^{\prime}\right)_{I}\right) \stackrel{\hat{\xi}_{*}^{\prime-1}}{\cong} H\left(\hat{A}_{I}^{\prime}\right) \stackrel{\hat{\rho}^{*}}{\longrightarrow} H\left(\hat{A}_{I}\right) \stackrel{\hat{\xi}_{*}}{\cong} H\left(W(g, H)_{I}\right) .
$$


3) Pour le calcul de $\operatorname{Ker} W(d \rho)^{*}$, on obtient un résultat analogue à celui du Corollaire 5.2.

\section{§6. Quelques exemples.}

Pour appliquer les résultats et techniques des paragraphes précédents et du travail [5], nous donnons dans ce paragraphe quelques exemples de sousfeuilletages localement homogènes $\left(F_{1}, F_{2}\right)=\left(F_{G_{1}}, F_{G_{2}}\right)$ de codimension $\left(q_{1}, q_{2}\right)$ sur $M=\Gamma \backslash \bar{G} / H$ ayant des classes caractéristiques secondaires ou exotiques non nulles qui n'appartiennent pas à la sous-algèbre de $H_{D R}(M)$, engendrée par les classes caractéristiques des feuilletages de la paire. De même, nous reprenons les notations des paragraphes précédents et de [5].

a) Exemples de sous-feuilletages localement homogènes dont les fibrés normaux $Q_{2}$ et $Q_{1} \oplus Q_{0}$ ne sont pas isomorphes comme fibrés vectoriels $F_{2}$-feuilletés (cf. [5], Corollaire 4.5).

Étant donnés des entiers $d \geqq 1$ et $j \geqq 0$, avec $j \leqq d$, on considère le sousgroupe fermé $G L(d+1, j) \subset G L(d+1)$ des matrices de la forme

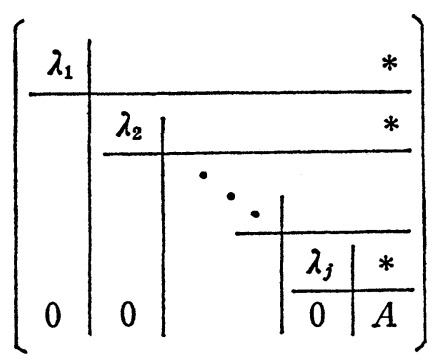

avec $\lambda_{1}, \lambda_{2}, \cdots, \lambda_{j} \in G L(1)$ et $A \in G L(d-j+1)$. En particulier, $S L(d+1, j)=$ $G L(d+1, j) \cap S L(d+1)$ est un sous-groupe fermé de $S L(d+1)$. On désigne par $G L^{+}(d+1, j)_{0}=G L(d+1, j)_{0}$ (resp. par $\left.S L(d+1, j)_{0}\right)$ la composante connexe du groupe $G L(d+1, j)$ (resp. du groupe $S L(d+1, j)$ ). Par conséquent, $S L(d+1, j)_{0}$ $\subset G L^{+}(d+1, j)_{0}$ pour $j=0,1, \cdots, d$. De même, on a donc le diagramme commutatif suivant:

$$
\begin{aligned}
& T(d+1)_{0}=G L^{+}(d+1, d)_{0} \subset \cdots \subset G L^{+}(d+1,1)_{0} \subset G L^{+}(d+1,0)_{0}=G L^{+}(d+1) \\
& \cap \cap \cap \cap \\
& T(d+1)=G L(d+1, d) \mid \subset \cdots \subset G L(d+1,1) \subset G L(d+1,0)=G L(d+1) \\
& \cup \quad \cup \quad \cup \quad \cup \quad U \\
& S T(d+1)=S L(d+1, d) \subset \cdots \subset S L(d+1,1) \subset S L(d+1,0)=S L(d+1) \\
& \cup \quad U \quad U \quad U \quad \| \\
& S T(d+1)_{0}=S L(d+1, d)_{0} \subset \cdots \subset S L(d+1,1)_{0} \subset S L(d+1,0)_{0}=S L(d+1),
\end{aligned}
$$


où $T(d+1)_{0}$ (resp. $\left.S T(d+1)_{0}\right)$ est la composante connexe du groupe $T(d+1)$ des matrices triangulaires (resp. du groupe $S T(d+1)$ des matrices triangulaires de déterminant 1).

Exemple 1. Prenons $\bar{G}=S L(d+2), G_{1}=S L(d+2,1)_{0}, G_{2}=S L(d+2,2)_{0} \quad H=$ $S O(d)$ (avec $d \geqq 1$ ). Soit $\Gamma \subset S L(d+2)$ un sous-groupe discret, uniforme et sans torsion. Alors, en vertu du Théorème 3.2, $M=\Gamma \backslash \bar{G} / H$ est une variété compacte connexe et orientable, et l'homomorphisme canonique $\gamma_{*}: H(\bar{g}, H) \rightarrow H_{D R}(M)$ est injectif. De plus, on a donc

$$
H(\bar{g}, H) \cong \begin{cases}\Lambda\left(\bar{y}_{3}, \bar{y}_{5}, \cdots, \bar{y}_{2 n-1}, \bar{y}_{d+1}, \bar{y}_{d+2}\right) & \text { pour } d=2 n-1 \\ \Lambda\left(\bar{y}_{3}, \bar{y}_{5}, \cdots, \bar{y}_{2 n-1}, \bar{y}_{d+1}, \bar{y}_{d+2}\right) \otimes R\left[e_{n}\right] /\left(e_{n}^{2}\right) & \text { pour } d=2 n,\end{cases}
$$

où les $\bar{y}_{i}$ sont les suspensions relatives des polynômes de Chern $\bar{c}_{i} \in \operatorname{Ker}$ res $=$ Idéal $\left\{\bar{c}_{3}, \bar{c}_{5}, \cdots, \bar{c}_{2 n-1}, \bar{c}_{d+1}, \bar{c}_{d+2}\right\} \subset I(\bar{G}) \cong R\left[\bar{c}_{2}, \bar{c}_{3}, \cdots, \bar{c}_{d+2}\right]$, et où $e_{n} \in I^{2 n}(S O(2 n))$ est le polynôme de Pfaff.

D'autre part, considérons le sous-feuilletage localement homogène $\left(F_{1}, F_{2}\right)=$ $\left(F_{G_{1}}, F_{G_{2}}\right)$ de codimension $\left(q_{1}, q_{2}\right)=(d+1,2 d+1)$ sur $M$ à fibré normal orienté. Nous obtenons alors le résultat suivant.

THÉORÈME 6.1. L'homomorphisme caractéristique

$$
\Delta_{*}: H\left(W(g l(d+1) \oplus g l(d), S O(d+1) \times S O(d))_{I}\right) \rightarrow H_{D R}(M)
$$

de ce sous-feuilletage vérifie les propriétés suivantes:

(i) Une R-base de $\operatorname{Im} \Delta_{*} \subset H_{D R}(M)$ est fournie par les classes linéairement indépendantes suivantes:

$$
\begin{aligned}
& \Delta_{*}\left[y_{1} \wedge y_{2 i_{1}-1} \wedge \cdots \wedge y_{2 i_{s}-1} \otimes c_{1}^{d+1} \otimes 1 \otimes 1 \otimes \Phi\right]=\mu \cdot \gamma_{*}\left(\bar{y}_{2 i_{1}-1} \wedge \cdots \wedge \bar{y}_{2^{i_{s}-1}} \wedge \bar{y}_{d+2} \otimes \Phi\right), \\
& \Delta_{*}\left[y_{1} \wedge y_{2 i_{1}-1} \wedge \cdots \wedge y_{2 i_{s}-1} \otimes c_{1}^{d+1} \otimes y_{1}^{\prime} \otimes c_{1}^{\prime d} \otimes \Phi\right] \\
& \quad=\kappa \cdot \gamma_{*}\left(\bar{y}_{2 i_{1}-1} \wedge \cdots \wedge \bar{y}_{2 i_{s}-1} \wedge \bar{y}_{d+1} \wedge \bar{y}_{d+2} \otimes \Phi\right) \text { et } \Delta_{*}[1 \otimes 1 \otimes 1 \otimes 1 \otimes \Phi]=\gamma_{*}(1 \otimes \Phi)
\end{aligned}
$$

pour $2 \leqq i_{1}<\cdots<i_{s} \leqq n, 0 \leqq s \leqq n-1$ (avec $s=0$ pour $n=1$ ), où $\mu=(-1)^{s+1}(d+2)^{d+2}$ et $\kappa=(-1)^{s+1}(d+2)^{d+2}(d+1)^{d+1}$, et où $\Phi=1$ si $d=2 n-1$, et $\Phi=1$ ou $\Phi=e_{n}$ si $d=2 n$. De plus, les éléments $\gamma_{*}(1 \otimes \Phi)$ fournissent une $R$-base des classes caractéristiques primaires de la $S O(d)$-réduction $\Gamma \backslash S L(d+2) \rightarrow M$ de $L\left(Q_{0}\right)$.

(ii) Pour $d=2 n-1$, on obtient

$\operatorname{Im} \Delta_{*}^{+}=\gamma_{*}\left(\bar{y}_{d+2} \cdot \Lambda\left(\bar{y}_{3}, \bar{y}_{5}, \cdots, \bar{y}_{2 n-1}, \bar{y}_{d+1}\right)\right), \operatorname{Im} \Delta_{*}^{+}\left(Q_{1}\right)=\gamma_{*}\left(\bar{y}_{d+2} \cdot \Lambda\left(\bar{y}_{3}, \bar{y}_{5}, \cdots, \bar{y}_{2 n-1}\right)\right)$,

$\operatorname{Im} \Delta_{* F_{2}}^{+}=\gamma_{*}\left(\left(\bar{y}_{d+2} \wedge \bar{y}_{d+1}\right) \cdot \Lambda\left(\bar{y}_{3}, \bar{y}_{5}, \cdots, \bar{y}_{2 n-1}\right)\right)$ et $\operatorname{Im} \Delta_{*}^{+}\left(Q_{2}\right)=\operatorname{Im} \Delta_{*}^{+}\left(Q_{0}\right)=0$;

par conséquent, $\operatorname{dim} \operatorname{Im} \Delta_{*}^{+}=2^{n}$ et $\operatorname{dim} \operatorname{Im} \Delta_{*}^{+}\left(Q_{1}\right)=\operatorname{dim} \operatorname{Im} \Delta_{* F_{2}}^{+}=2^{n-1}$. Il en résulte que

$$
0=\operatorname{Im} \Delta_{*}^{+}\left(Q_{2}\right)=\operatorname{Im} \Delta_{*}^{+}\left(Q_{0}\right) \subsetneq \operatorname{Im} \Delta_{*}^{+}\left(Q_{1}\right) \subsetneq \operatorname{Im} \Delta_{*}^{+}=\operatorname{Im} \Delta_{*}^{+}\left(Q_{1}\right) \oplus \operatorname{Im} \Delta_{* F_{2}}^{+}
$$


(iii) Pour $d=2 n$, on a donc

$\operatorname{Im} \Delta_{*}^{+}=\gamma_{*}\left(\bar{y}_{d+2} \cdot \Lambda\left(\bar{y}_{3}, \bar{y}_{5}, \cdots, \bar{y}_{2 n-1}, \bar{y}_{d+1}\right) \otimes R\left[e_{n}\right] /\left(e_{n}^{2}\right) \oplus 1 \otimes R\left[e_{n}\right]^{+} /\left(e_{n}^{2}\right)\right)$,

$\operatorname{Im} \Delta_{*}^{+}\left(Q_{1}\right)=\gamma_{*}\left(\bar{y}_{d+2} \cdot \Lambda\left(\bar{y}_{3}, \bar{y}_{5}, \cdots, \bar{y}_{2 n-1}, \bar{y}_{d+1}\right)\right)$,

$\operatorname{Im} \Delta_{* F_{2}}^{+}=\gamma_{*}\left(\left(\bar{y}_{d+2} \wedge \bar{y}_{d+1}\right) \cdot \Lambda\left(\bar{y}_{3}, \bar{y}_{5}, \cdots, \bar{y}_{2 n-1}\right) \otimes R\left[e_{n}\right] /\left(e_{n}^{2}\right) \oplus 1 \otimes R\left[e_{n}\right]^{+} /\left(e_{n}^{2}\right)\right)$,

$\operatorname{Im} \Delta_{*}^{+}\left(Q_{0}\right)=\gamma_{*}\left(1 \otimes R\left[e_{n}\right]^{+} /\left(e_{n}^{2}\right)\right)$ et $\operatorname{Im} \Delta_{*}^{+}\left(Q_{2}\right)=0$;

par suite, $\operatorname{dim} \operatorname{Im} \Delta_{*}^{+}=2^{n+1}+1, \quad \operatorname{dim} \operatorname{Im} \Delta_{*}^{+}\left(Q_{1}\right)=2^{n}, \quad \operatorname{dim} \operatorname{Im} \Delta_{* F_{2}}^{+}=2^{n}+1 \quad$ et $\operatorname{dim} \operatorname{Im} \Delta_{*}^{+}\left(Q_{0}\right)=1$. Il s'ensuit que

$$
0=\operatorname{Im} \Delta_{*}^{+}\left(Q_{2}\right) \subsetneq \operatorname{Im} \Delta_{*}^{+}\left(Q_{0}\right) \subsetneq \operatorname{Im} \Delta_{* F_{2}}^{+} \subsetneq \operatorname{Im} \Delta_{*}^{+}\left(Q_{1}\right)+\operatorname{Im} \Delta_{* F_{2}}^{+} \text {Im } \Delta_{*}^{+} .
$$

(iv) L'ensemble $N_{\left(F_{1}, F_{2}\right)} \subset \operatorname{Im} \Delta_{*}^{+}$des classes caractéristiques secondaires non nulles de $\left(F_{1}, F_{2}\right)$, qui n'appartiennent pas à la sous-algèbre de $H_{D R}(M)$, engendrée par les classes caractéristiques des feuilletages de la paire, est donné par

$$
N_{\left(F_{1}, F_{2}\right)}=\left\{\begin{array}{l}
\gamma_{*}\left(\left(\bar{y}_{d+2} \wedge \bar{y}_{d+1}\right) \cdot \Lambda\left(\bar{y}_{3}, \bar{y}_{5}, \cdots, \bar{y}_{2 n-1}\right)\right)-\{0\} \quad \text { pour } d=2 n-1 \\
\gamma_{*}\left(\bar{y}_{d+2} \cdot \Lambda\left(\bar{y}_{3}, \bar{y}_{5}, \cdots, \bar{y}_{2 n-1}, \bar{y}_{d+1}\right) \otimes R\left[e_{n}\right]^{+} /\left(e_{n}^{2}\right)\right)-\{0\} \text { pour } d=2 n .
\end{array}\right.
$$

Par conséquent, ces classes secondaires engendrent un sous-espace vectoriel de $H_{D R}(M)$ de dimension $2^{[d / 2]}$.

(v) Pour certaines classes de Godbillon-Vey de ce sous-feuilletage, on a la formule

$$
\Delta_{*}\left[y_{1} \otimes c_{1}^{d+1-k} \otimes y_{1}^{\prime} \otimes c_{1}^{\prime d+k}\right]=(d+2)^{d+2-k}(d+1)^{d+1}(k-1)\left(\begin{array}{c}
d+k \\
d
\end{array}\right) \gamma_{*}\left(\bar{y}_{d+1} \wedge \bar{y}_{d+2}\right)
$$

pour $k=0,1, \cdots, d+1$. Puisque, pour $k \neq 1$ et $0 \leqq k \leqq d+1$, les classes précédentes ne sont pas nulles, le Corollaire 4.5 dans [5] montre que $Q_{2}$ et $Q_{1} \oplus Q_{0}$ ne sont pas isomorphes comme fibrés vectoriels $F_{2}$-feuilletés. De même, on vérifie que la structure $\left(F_{1}, F_{2}\right)$-feuilletée de $\bar{P}=L\left(Q_{1}\right)+L\left(Q_{0}\right)$ n'est pas induite par une structure $\left(F_{1}, F_{2}\right)$-feuilletée d'une $S L\left(q_{1}\right) \times S L(d)$-réduction de $\bar{P}$, et que les sous-algèbres de Lie $g_{1}$ et $g_{2}$ ne sont pas réductives dans $\bar{g}$.

(vi) Les autres classes de Godbillon-Vey de $\left(F_{1}, F_{2}\right)$ sont données par

$$
\begin{aligned}
& \Delta_{*}\left(Q_{1}\right)\left[y_{1} \otimes c_{1}^{d+1}\right]=\Delta_{*}\left[y_{1} \otimes c_{1}^{d+1} \otimes 1 \otimes 1\right]=-(d+2)^{d+2} \gamma *\left(\bar{y}_{d+2}\right) \neq 0, \\
& \Delta_{*}\left[1 \otimes c_{1}^{d+1-k} \otimes y_{1}^{\prime} \otimes c_{1}^{d+k}\right]=0 \text { pour } k=0,1, \cdots, d+1 .
\end{aligned}
$$

(vii) Pour $d=2 n$, la classe

$$
\Delta_{*}\left(Q_{1}\right)\left[y_{1} \wedge y_{d+1} \otimes c_{1}^{d+1}\right]=(d+2)^{d+2} \gamma_{*}\left(\bar{y}_{d+1} \wedge \bar{y}_{d+2}\right)
$$

n'est pas nulle. Par suite, d'après le Corollaire 5.4, aucun feuilletage intégrablement homotope à $F_{1}$ ne peut se prolonger en un feuilletage de codimension $q$, avec $0<q<q_{1}=2 n+1$. 
(viii) Les classes rigides appartiennent à $\operatorname{Ker} \Delta_{*}$.

(ix) Les anneaux de Pontrjagin de $Q_{i}, i=0,1,2$, et $Q_{1} \oplus Q_{0}$ sont triviaux.

Démonstration. Considérons la paire de groupes de Lie $\left(G_{1}^{\prime}, G_{2}^{\prime}\right)=\left(G L^{+}(d+1)\right.$, $\left.G L^{+}(1) \times G L^{+}(d)\right)$ et sa paire d'algèbres de Lie $\left(g_{1}^{\prime}, g_{2}^{\prime}\right)=(g l(d+1), g l(1) \oplus g l(d))$. Soient $\pi=\left(\pi_{1}, \pi_{2}\right): G_{1} \times G_{2} \rightarrow G_{1}^{\prime} \times G_{2}^{\prime}$ la projection canonique et $\rho^{\prime}=\left(\rho_{1}^{\prime}, \rho_{0}^{\prime}\right): G_{1}^{\prime} \times G_{2}^{\prime}$ $\rightarrow G L^{+}(d+1) \times G L^{+}(d)$ l'homomorphisme de groupes de Lie défini par

$$
\rho^{\prime}(A,(\lambda, B))=\left(\rho_{1}^{\prime}(A), \rho_{0}^{\prime}(\lambda, B)\right)=\left(\text { dét } A \cdot A, \lambda^{-1} \cdot B\right) \text { pour }(A,(\lambda, B)) \in G_{1}^{\prime} \times G_{2}^{\prime} .
$$

Il est immédiat de voir que la représentation d'isotropie

$$
\rho=\left(\rho_{1}, \rho_{0}\right): G_{1} \times G_{2} \rightarrow G L^{+}\left(m_{1}\right) \times G L^{+}\left(m_{0}\right) \cong G L^{+}(d+1) \times G L^{+}(d)
$$

se factorise de la manière suivante:

$$
\rho=\rho^{\prime} \circ \pi: G_{1} \times G_{2} \rightarrow G_{1}^{\prime} \times G_{2}^{\prime} \rightarrow G L^{+}(d+1) \times G L^{+}(d) .
$$

Considérons maintenant, pour chaque $i=1,2$, une scission $G_{i}^{\prime}$-équivariante $\theta_{i}: \bar{g} \rightarrow g_{i}$ de la suite exacte $0 \rightarrow g_{i} \rightarrow \bar{g} \rightarrow m_{i} \cong R^{q_{i} \rightarrow 0}$. Il s'ensuit que, pour $i=1,2$, l'application composée $\theta_{i}^{\prime}=d \pi_{i} \circ \theta_{i}: \bar{g} \rightarrow g_{i}^{\prime}$ est une scission $G_{i}^{\prime}$-équivariante de la

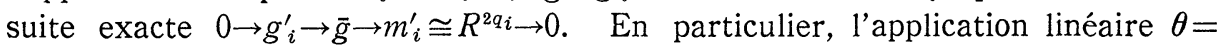
$\theta_{1}+\theta_{2}: \bar{g} \rightarrow g_{1} \oplus g_{2}$ détermine une connexion adaptée somme sur le fibré principal $\left(F_{1}, F_{2}\right)$-feuilleté localement homogène

$$
P=P_{1}+P_{2}=\Gamma \backslash \bar{G} \times{ }_{H} G_{1}+\Gamma \backslash \bar{G} \times{ }_{H} G_{2}=\Gamma \backslash \bar{G} \times{ }_{H}\left(G_{1} \times G_{2}\right) \rightarrow M .
$$

De même, l'application linéaire $\theta^{\prime}=d \pi \circ \theta=\theta_{1}^{\prime}+\theta_{2}^{\prime}: \bar{g} \rightarrow g_{1}^{\prime} \oplus g_{2}^{\prime}$ induit une connexion basique somme sur le fibré principal $\left(F_{G_{1}^{\prime}}, F_{G_{2}^{\prime}}\right)$-feuilleté localement homogène

$$
P^{\prime}=P_{1}^{\prime}+P_{2}^{\prime}=\Gamma \backslash \bar{G} \times{ }_{H} G_{1}^{\prime}+\Gamma \backslash \bar{G} \times{ }_{H} G_{2}^{\prime}=\Gamma \backslash \bar{G} \times{ }_{H}\left(G_{1}^{\prime} \times G_{2}^{\prime}\right) \rightarrow M .
$$

Il en résulte que l'homomorphisme caractéristique $\Delta_{*}$ du sous-feuilletage $\left(F_{1}, F_{2}\right)$ est donné par la composition où

$$
\Delta_{*}=\Delta_{*}\left(P^{\prime}\right) \circ W\left(d \rho^{\prime}\right)^{*}: H\left(W(g l(d+1) \oplus g l(d), S O(d+1) \times S O(d))_{I}\right) \rightarrow H_{D R}(M),
$$

$$
\Delta_{*}\left(P^{\prime}\right)=\Delta_{*}(P) \circ W(d \pi)^{*}=\gamma_{*}^{\circ} \Delta\left(\theta^{\prime}\right)_{*}: H\left(W\left(g_{1}^{\prime} \oplus g_{2}^{\prime}, H \times H\right)_{I^{\prime \prime}}\right) \rightarrow H(\bar{g}, H) \rightarrow H_{D R}(M)
$$

est l'homomorphisme caractéristique généralisé du fibré principal $\left(F_{G_{1}^{\prime}}, F_{G_{2}^{\prime}}\right)$ feuilleté localement homogène $P^{\prime}=P_{1}^{\prime}+P_{2}^{\prime}$ (à connexion basique somme déterminée $\left.\operatorname{par} \theta^{\prime}=d \pi \circ \theta=\theta_{1}^{\prime}+\theta_{2}^{\prime}\right)$.

D'apprès le Théorème 4.3 , pour calculer l'homomorphisme $\Delta\left(\theta^{\prime}\right)_{*}$, il faut d'abord considérer les applications de restrictions

$$
\begin{aligned}
& i_{2}^{*}: I(S L(d+2)) \stackrel{i_{1}^{*}}{\longrightarrow} I(G L(d+1)) \stackrel{i_{0}^{*}}{\longrightarrow} I(G L(1) \times G L(d)) \\
& \mathbb{R} \quad \mathbb{R} \quad \mathbb{R} \\
& R\left[\bar{c}_{2}, \cdots, \bar{c}_{d+2}\right] \longrightarrow R\left[c_{1}, \cdots, c_{d+1}\right] \longrightarrow R\left[c_{1}^{\prime \prime}\right] \otimes R\left[c_{1}^{\prime}, \cdots, c_{d}^{\prime}\right]
\end{aligned}
$$


données respectivement, pour $j=1, \cdots, d+1$, par les formules suivantes:

$$
\begin{aligned}
& i_{1}^{*} \bar{c}_{j+1}=c_{j+1}-c_{1} c_{\jmath} \text { avec } c_{d+2}=0, \\
& i_{0}^{*} c_{\jmath}=c_{\jmath}^{\prime}+c_{1}^{\prime \prime} c_{j-1}^{\prime} \text { avec } c_{0}^{\prime}=1 \text { et } c_{d+1}^{\prime}=0, \\
& i_{2}^{*} \bar{c}_{j+1}=c_{j+1}^{\prime}-c_{1}^{\prime} c_{j}^{\prime}-c_{1}^{\prime \prime} c_{1}^{\prime} c_{j-1}^{\prime}-c_{1}^{\prime \prime 2} c_{\jmath-1}^{\prime} \text { avec } c_{0}^{\prime}=1 \text { et } c_{d+1}^{\prime}=c_{d+2}^{\prime}=0,
\end{aligned}
$$

où les $\bar{c}_{j}, c_{j}, c_{j}^{\prime}$ et $c_{1}^{\prime \prime}$ sont les polynômes de Chern. Par conséquent, $\bar{c}_{d+2}$ et $\bar{c}_{d+1}$ sont les deux générateurs pour appliquer le Théorème 4.3. De même, pour utiliser la Proposition 4.2, considérons les congruités suivantes $\bmod I^{+}(S L(d+2))$. $I(G L(d+1))$ :

$$
c_{\jmath} \equiv c_{1}^{\jmath} \text { pour } j=1, \cdots, d+1, \quad c_{1}^{d+2} \equiv c_{1} c_{d+1} \equiv 0,
$$

et les congruités correspondantes $\bmod I^{+}(S L(d+2)) \cdot I(G L(1) \times G L(d)): c_{j}^{\prime} \equiv$ $\sum_{k=0}^{j}(-1)^{k} c_{1}^{\prime \prime k}\left(c_{1}^{\prime}+c_{1}^{\prime \prime}\right)^{j-k} \equiv\left(c_{1}^{\prime}+c_{1}^{\prime \prime}\right) c_{j-1}^{\prime}+(-1)^{j} c_{1}^{\prime \prime}$ pour $j=1, \cdots d+1 \quad$ (avec $\left.c_{d+1}^{\prime}=0\right)$, $c_{1}^{\prime d+t} \equiv c_{1}^{\prime \prime t}\left(\sum_{k=0}^{d} \alpha_{t k} c_{1}^{\prime \prime k} c_{1}^{\prime d-k}\right)$ pour $t \geqq 1$, où les $\alpha_{t k}$ sont des entiers, $c_{1}^{\prime \prime d+t} \equiv\left(c_{1}^{\prime}+c_{1}^{\prime \prime}\right)^{d+t}$ $\equiv 0$ pour $t \geqq 2$, et $\left(c_{1}^{\prime}+c_{1}^{\prime \prime}\right)^{d+1} c_{1}^{\prime \prime d} \equiv\left(c_{1}^{\prime}+c_{1}^{\prime \prime}\right)^{d} c_{1}^{\prime \prime d+1} \equiv(-1)^{d}\left(c_{1}^{\prime}+c_{1}^{\prime \prime}\right) c_{d}^{\prime 2} \equiv c_{1}^{\prime \prime d}{ }^{d+1} c_{1}^{\prime d}$.

D'autre part, pour le calcul de l'homomorphisme $W\left(d \rho^{\prime}\right)^{*}$, d'après le Théorème 5.5 , il faut considérer l'homomorphisme

$$
\begin{aligned}
\rho^{\prime *}=\rho_{1}^{\prime *} \otimes \rho_{0}^{*}: I(G L(d+1)) \otimes I(G L(d)) & \longrightarrow I(G L(d+1)) \otimes I(G L(1) \times G L(d)) \\
R\left[c_{1}, \cdots, c_{d+1}\right] \otimes R\left[c_{1}^{\prime}, \cdots, c_{d}^{\prime}\right] & \longrightarrow R\left[c_{1}, \cdots, c_{d+1}\right] \otimes\left(R\left[c_{1}^{\prime \prime}\right] \otimes R\left[c_{1}^{\prime}, \cdots, c_{d}^{\prime}\right]\right)
\end{aligned}
$$

défini par les formules suivantes:

$$
\begin{aligned}
& \rho_{1}^{\prime *} c_{j}=\sum_{k=0}^{j}\left(\begin{array}{c}
d+1-k \\
j-k
\end{array}\right) c_{1}^{j-k} c_{k} \text { pour } j=1, \cdots, d+1\left(\text { avec } c_{0}=1\right), \\
& \rho_{0}^{\prime *} c_{j}^{\prime}=\sum_{k=0}^{j}(-1)^{j-k}\left(\begin{array}{c}
d-k \\
j-k
\end{array}\right) c_{1}^{\prime \prime j-k} c_{k}^{\prime} \text { pour } j=1, \cdots, d\left(\text { avec } c_{0}^{\prime}=1\right) .
\end{aligned}
$$

Par suite, on a alors

$$
\begin{aligned}
& \hat{\rho}_{1}^{\prime}\left(y_{j}\right)=y_{j} \otimes 1+y_{1} \otimes\left(\sum_{k=0}^{j-1}\left(\begin{array}{c}
d+1-k \\
j-k
\end{array}\right) c_{1}^{j-k-1} c_{k}\right) \text { pour } j \text { impair et } 1 \leqq j \leqq d+1, \\
& \hat{\rho}_{0}^{\prime}\left(y_{j}^{\prime}\right)=y_{j}^{\prime} \otimes 1+y_{1}^{\prime \prime} \otimes\left(\sum_{k=0}^{j-1}(-1)^{j-k}\left(\begin{array}{c}
d-k \\
j-k
\end{array}\right) c_{1}^{\prime \prime-k-1} c_{k}^{\prime}\right) \text { pour } j \text { impair et } 1 \leqq j \leqq d,
\end{aligned}
$$

où les $y_{j}, y_{j}^{\prime}$ et $y_{1}^{\prime \prime}$ sont respectivement les suspensions relatives des polynômes de Chern $c_{j}, c_{j}^{\prime}$ et $c_{1}^{\prime \prime}$, avec $j$ impair.

Compte tenu des Propositions 2.6 et 2.8, on obtient donc

$$
\operatorname{Im} \Delta_{*}=\operatorname{Im} \Delta_{*}\left(P^{\prime}\right)=\operatorname{Im} \Delta_{*}\left(P_{1}^{\prime}\right) \text { et } \operatorname{Im} \Delta_{* F_{2}}=\operatorname{Im} \Delta_{*}\left(P_{2}^{\prime}\right) \text {. }
$$

Le théorème en résulte. 
Remarques. 1) Pour $\Phi=1$ si $d=2 n-1$ et $\Phi=e_{n}$ si $d=2 n$, le volume

$$
\Delta_{*}\left[y_{1} \wedge y_{3} \wedge \cdots \wedge y_{2 n-1} \otimes c_{1}^{d+1} \otimes y_{1}^{\prime} \otimes c_{1}^{\prime d} \otimes \Phi\right]=\kappa \cdot \gamma_{*}\left(\bar{y}_{3} \wedge \cdots \wedge \bar{y}_{2 n-1} \wedge \bar{y}_{d+1} \wedge \bar{y}_{d+2} \otimes \Phi\right)
$$

de $M$, avec $\kappa=(-1)^{n}(d+2)^{d+2}(d+1)^{d+1}$, est un invariant non nul du sous-feuilletage $\left(F_{1}, F_{2}\right)$, qui n'appartient pas à la sous-algèbre de $H_{D R}(M)$ engendrée par les classes caractéristiques des feuilletages de la paire. De même, pour $d=2 n-1$, l'un de ces invariants est la classe de Godbillon-Vey

$$
\Delta_{*}\left[y_{1} \otimes c_{1}^{d+1} \otimes y_{1}^{\prime} \otimes c_{1}^{\prime d}\right]=-(d+2)^{d+2}(d+1)^{d+1} \cdot \gamma_{*}\left(\bar{y}_{d+1} \wedge \bar{y}_{d+2}\right) \neq 0 .
$$

2) Le Théorème 6.1 est une généralisation du Théorème 5.37 (parties (i), (ii), (iii), (iv) et (vii)) dans [11]. En particulier, l'Exemple 1 est une généralisation de l'exemple de Roussarie [7].

Exemple 2. Prenons $\bar{G}=S L(d+2), G_{1}=S L(d+2,1), G_{2}=S L(d+2,2), \quad H=$ $O(d)$ et $\Gamma \subset S L(d+2)$ un sous-groupe discret, uniforme et sans torsion. Considérons le sous-feuilletage localement homogène $\left(F_{1}, F_{2}\right)=\left(F_{G_{1}}, F_{G_{2}}\right)$ de codimension $\left(q_{1}, q_{2}\right)=(d+1,2 d+1)$ sur $M=\Gamma \backslash \bar{G} / H$ (dont le fibré normal n'est pas nécessairement orientable). En oubliant maintenant la structure orientée du fibré normal du sous-feuilletage donné dans l'Exemple 1, on déduit du Théorème 6.1 et de la Proposition 2.10 le résultat suivant.

THÉORÈME 6.2. L'homomorphisme caractéristique $\Delta_{*}: H\left(W O_{I}\right) \rightarrow H_{D R}(M)$ de ce sous-feuilletage est donné par le Théorème 6.1, en remplaçant le polynôme de Pfaff $e_{n}$ par 0 pour $d=2 n$. Par suite, on vérifie les propriétés suivantes:

(i) Pour $d=2 n-1$, on a donc

$$
0=\operatorname{Im} \Delta_{*}^{+}\left(Q_{2}\right)=\operatorname{Im} \Delta_{*}^{+}\left(Q_{0}\right) \subsetneq \operatorname{Im} \Delta_{*}^{+}\left(Q_{1}\right) \subsetneq \operatorname{Im} \Delta_{*}^{+}=\operatorname{Im} \Delta_{*}^{+}\left(Q_{1}\right) \oplus \operatorname{Im} \Delta_{* F_{2}}^{+}
$$

avec

$\operatorname{Im} \Delta_{*}^{+}=\gamma_{*}\left(\bar{y}_{d+2} \cdot \Lambda\left(\bar{y}_{3}, \bar{y}_{5}, \cdots, \bar{y}_{2 n-1}, \bar{y}_{d+1}\right)\right), \operatorname{Im} \Delta_{*}^{+}\left(Q_{1}\right)=\gamma_{*}\left(\bar{y}_{d+2} \cdot \Lambda\left(\bar{y}_{3}, \bar{y}_{5}, \cdots, \bar{y}_{2 n-1}\right)\right)$ et $\operatorname{Im} \Delta_{* F_{2}}^{+}=\gamma_{*}\left(\left(\bar{y}_{d+2} \wedge \bar{y}_{d+1}\right) \cdot \Lambda \wedge\left(\bar{y}_{3}, \bar{y}_{5}, \cdots, \bar{y}_{2 n-1}\right)\right) ;$ par conséquent, $\operatorname{dim} \operatorname{Im} \Delta_{*}^{+}=2^{n}$ et $\operatorname{dim} \operatorname{Im} \Delta_{*}^{+}\left(Q_{1}\right)=\operatorname{dim} \operatorname{Im} \Delta_{* F_{2}}^{+}=2^{n-1}$.

(ii) Pour $d=2 n$, on obtient

$$
0=\operatorname{Im} \Delta_{*}^{+}\left(Q_{2}\right)=\operatorname{Im} \Delta_{*}^{+}\left(Q_{0}\right) \subsetneq \operatorname{Im} \Delta_{*}^{+}\left(Q_{1}\right)=\operatorname{Im} \Delta_{*}^{+}
$$

avec $\operatorname{Im} \Delta_{*}^{+}=\gamma_{*}\left(\bar{y}_{d+2} \cdot \Lambda\left(\bar{y}_{3}, \bar{y}_{5}, \cdots, \bar{y}_{2 n-1}, \bar{y}_{d+1}\right)\right)$ et $\operatorname{Im} \Delta_{* F_{2}}^{+}=\gamma_{*}\left(\left(\bar{y}_{d+2} \wedge \bar{y}_{d+1}\right)\right.$. $\left.\Lambda\left(\bar{y}_{3}, \bar{y}_{5}, \cdots, \bar{y}_{2 n-1}\right)\right) ;$ par suite, $\operatorname{dim} \operatorname{Im} \Delta_{*}^{+}=\operatorname{dim} \operatorname{Im} \Delta_{*}^{+}\left(Q_{1}\right)=2^{n}$ et $\operatorname{dim} \operatorname{Im} \Delta_{* F_{2}}^{+}=2^{n-1}$.

(iii) Pour $d=2 n-1$, il en résulte que

$$
N_{\left(F_{1}, F_{2}\right)}=\gamma_{*}\left(\left(\bar{y}_{d+2} \wedge \bar{y}_{d+1}\right) \cdot \Lambda\left(\bar{y}_{3}, \bar{y}_{5}, \cdots, \bar{y}_{2 n-1}\right)\right)-\{0\}
$$

est l'ensemble des classes caractéristiques secondaires non nulles de $\left(F_{1}, F_{2}\right)$, qui n'appartiennent pas à la sous-algèbre de $H_{D R}(M)$ engendrée par les classes caractéristiques des feuilletages de la paire.

(iv) $Q_{2}$ et $Q_{1} \oplus Q_{0}$ ne sont pas isomorphes comme fibrés vectoriels $F_{2}$-feuilletés.

(v) Aucun feuilletage intégrablement homotope à $F_{1}$ ne peut se prolonger en 
un feuilletage de codimension $q$ pour $0<q<q_{1}=2 n+1$.

(vi) Les classes rigides appartiennent à Ker $\Delta_{*}$.

(vii) Les anneaux de Pontrjagin de $Q_{i}, i=0,1,2$, et $Q_{1} \oplus Q_{0}$ sont triviaux.

On peut maintenant discuter l'exemple suivant.

Exemple 3. Pour $d \geqq 1$ et $0 \leqq j \leqq d-1$, considérons les sous-feuilletages localement homogènes de codimension $\left(q_{1}, q_{2}\right)=(d-j+1,2(d-j)+1)$ induits par les groupes de Lie correspondants aux quatre cas suivants:

i) $\bar{G}=S L(d+2, j)_{0}, \quad G_{1}=S L(d+2, j+1)_{0}, \quad G_{2}=S L(d+2, j+2)_{0}, \quad H=S O(d-j)$ et $\Gamma$ un sous-groupe discret, uniforme et sans torsion de $S L(d+2)$ (resp. de $G L^{+}(d-j+2)$ ) pour $j=0$ (resp. pour $\left.j>0\right)$.

ii) $\bar{G}=S L(d+2, j), G_{1}=S L(d+2, j+1), G_{2}=S L(d+2, j+2), H=O(d-j)$ et $\Gamma$ le groupe donné dans le cas i).

iii) $\bar{G}=G L^{+}(d+2, j)_{0}, \quad G_{1}=G L^{+}(d+2, j+1)_{0}, \quad G_{2}=G L^{+}(d+2, j+2)_{0}, \quad H=$ $S O(d-j)$ et $\Gamma \subset G L^{+}(d-j+2)$ un sous-groupe discret, uniforme et sans torsion.

iv) $\bar{G}=G L(d+2, j), \quad G_{1}=G L(d+2, j+1), \quad G_{2}=G L(d+2, j+2), \quad H=O(d-j)$ et $\Gamma$ le groupe considéré dans le cas iii).

Il est clair que les fibrés normaux des sous-feuilletages localement homogènes correspondants aux cas i) et iii) sont orientés; de plus, on déduit du Théorème 6.1 et de la Proposition 2.10 que les classes caractéristiques de ces sous-feuilletages sont données par le Théorème 6.1 en remplaçant $d \geqq 1$ par $d-j \geqq 1$. De même, les classes caractéristiques des sous-feuilletages localement homogènes correspondants aux cas ii) et iv) sont données par le Théorème 6.2 (en remplaçant $d \geqq 1$ par $d-j \geqq 1$ ). Par suite, pour les cas i) et iii) (resp. pour les cas ii) et iv)), on vérifie les propriétés énoncées dans le Théorème 6.1 (resp. dans le Théorème 6.2). Il en résulte que, pour $d \geqq 1$ et $0 \leqq j \leqq d-1$, la sousalgèbre de Lie $s l(d+1, j+1)$ (resp. $g l(d+1, j+1)$ ) n'est pas réductive dans $s l(d+1, j)$ (resp. dans $g l(d+1, j))$. Puisque le centre $Z_{s l(d+1, j)}$ de $s l(d+1, j)$ est zéro (resp. le centre de $g l(d+1, j)$ est donné par $Z_{g l(d+1, j)}=R$. I, où I désigne la matrice identité), il s'ensuit que, pour $d \geqq 1$ et $1 \leqq j \leqq d$, l'algèbre de Lie $s l(d+1, j)$ (resp. $g l(d+1, j))$ n'est pas réductive.

D'autre part, si dans l'Exemple 3 (cas i), ii), iii) et iv)), les groupes de Lie $\bar{G}, G_{1}$ et $G_{2}$ sont remplacés respectivement par les groupes de Lie $\bar{G}^{t}$, $G_{1}^{t}$ et $G_{2}^{t}$ de leurs matrices transposées, alors les classes caractéristiques de ces sousfeuilletages localement homogènes sont données (à un signe près) par l'Exemple 3. En particulier, pour $j=0$, on a donc

$$
\Delta_{*}^{t}\left[y_{1} \otimes c_{1}^{d+1} \otimes y_{1}^{\prime} \otimes c_{1}^{\prime d}\right]=-\Delta_{*}\left[y_{1} \otimes c_{1}^{d+1} \otimes y_{1}^{\prime} \otimes c_{1}^{\prime d}\right] \neq 0 \in H_{D R}^{2 q_{2}+2}(M),
$$

où $\Delta_{*}^{t}$ (resp. $\Delta_{*}$ ) désigne l'homomorphisme caractéristique de chacun de ces sous-feuilletages (resp. de chacun des sous-feuilletages donnés dans l'Exemple 3), avec $j=0$.

On en déduit le 
COROLlatre 6.3. Il existe au moins deux sous-feuilletages de codimension $\left(q_{1}, q_{2}\right)=(d+1,2 d+1)$ sur la variété correspondante $M=\Gamma \backslash \bar{G} / H$, qui ne sont pas intégrablement homotopes.

b) Exemples de sous-feuilletages localement homogènes dont les fibrés normaux $Q_{2}$ et $Q_{1} \oplus Q_{0}$ sont isomorphes comme fibrés vectoriels $F_{2}$-feuilletés (cf. Corollaire 2.3).

Exemple 4. Pour les groupes de Lie compacts connexes $\bar{G}=S U(d+2), G_{1}=$ $U(d+1), G_{2}=U(1) \times U(d), H=S O(d)$ et $\Gamma=\{e\}$ (avec $d \geqq 1$ ), considérons le sousfeuilletage homogène $\left(F_{1}, F_{2}\right)=\left(F_{G_{1}}, F_{G_{2}}\right)$ de codimension $\left(q_{1}, q_{2}\right)=(2(d+1), 2(2 d+1))$ sur $M=S U(d+2) / S O(d)$, dont le fibré normal complexe $Q_{1} \oplus Q_{0}$ admet une connexion basique somme. Il est bien évident que l'homomorphisme caractéristique

$$
\Delta_{*}: H\left(W(u(d+1) \oplus u(d), S O(d+1) \times S O(d))_{I^{\prime \prime}}\right) \longrightarrow H(\bar{g}, H) \cong H_{D R}(M)
$$

de ce sous-feuilletage peut être calculé, pour $d=2 n-1$ et $d=2 n$, par les techniques du Théorème 6.1. De plus, les classes caractéristiques de $\left(F_{1}, F_{2}\right)$ sont données par ce théorème. Comme l'espace $(U(d+1) \times U(d)) /(S O(d+1) \times S O(d))$ n'est pas contractile, les classes caractéristiques précédentes ne dépendent que de la classe d'homotopie de la section $s: M \rightarrow\left(U\left(Q_{1}\right)+U\left(Q_{0}\right)\right) /(S O(d+1) \times S O(d))$ induite par la section canonique $S U(d+2) \rightarrow S U(d+2) \times(\{e\} \times\{e\})$ de $S U(d+2) \times$ $(U(d+1) \times(U(1) \times U(d)))$, où $U\left(Q_{1}\right)+U\left(Q_{0}\right)$ désigne le fibré des $U(d+1) \times U(d)$ repères de $Q_{1} \oplus Q_{0}$.

Remarque. Si dans l'Exemple 4, on remplace $H=S O(d)$ par $H=O(d)$, alors les classes caractéristiques d'un tel sous-feuilletage sont données par le Théorème 6.2 .

Exemple 5. Prenons $\bar{G}=S O(4), G_{1}=U(2), G_{2}=U(1) \times U(1)$ et $H=\Gamma=\{e\}$. Considérons le sous-feuilletage homogène $\left(F_{1}, F_{2}\right)=\left(F_{G_{1}}, F_{G_{2}}\right)$ de codimension $\left(q_{1}\right.$, $\left.q_{2}\right)=(2,4)$ sur $M=S O(4)$, dont le fibré normal complexe trivialisé $Q_{1} \oplus Q_{0}$ admet une connexion basique somme. Il est immédiat de voir que l'homomorphisme caractéristique

$$
\Delta_{*}: H\left(W(u(1) \oplus u(1))_{I^{\prime \prime}}\right) \cong H\left(W(s o(2) \oplus s o(2))_{I^{\prime \prime}}\right) \longrightarrow H(s o(4)) \cong H_{D R}(M)
$$

de ce sous-feuilletage vérifie les propriétés suivantes:

(i) Une base de Im $\Delta_{*}^{+} \subset H_{D R}^{+}(M) \cong \Lambda^{+}\left(\bar{y}_{1}, \bar{x}_{2}\right)$ est fournie par les deux classes linéairement indépendantes suivantes:

et

$$
\Delta_{*}\left[y_{1} \otimes c_{1} \otimes 1 \otimes 1\right]=\bar{y}_{1}+2 \bar{x}_{2} \in H_{D R}^{3}(M)
$$

$$
\Delta_{*}\left[y_{1} \otimes c_{1} \otimes y_{1}^{\prime} \otimes c_{1}^{\prime}\right]=-4 \bar{y}_{1} \wedge \bar{x}_{2} \in H_{D R}^{6}(M),
$$

où $y_{1}$ et $y_{1}^{\prime}$ sont respectivement les suspensions des polynômes de Chern $c_{1}$ et $c_{1}^{\prime}$ dans $I(U(1) \times U(1)) \cong R\left[c_{1}\right] \otimes R\left[c_{1}^{\prime}\right]$, et où $\bar{y}_{1}\left(\right.$ resp. $\left.\bar{x}_{2}\right)$ est la suspension $\mathrm{du}$ polynôme de Pontrjagin $\bar{p}_{1}$ (resp. du polynôme de Pfaff $\bar{e}_{2}$ ) dans $I(S O(4)) \cong R\left[\bar{p}_{1}, \bar{e}_{2}\right]$. 
(ii) Une base de $\operatorname{Im} \Delta_{*}^{+}\left(Q_{1}\right)$ (resp. de $\operatorname{Im} \Delta_{* F_{2}}^{+}$) est fournie par la classe $\bar{y}_{1}+$ $2 \bar{x}_{2}$ (resp. par la classe $\bar{y}_{1} \wedge \bar{x}_{2}$ ). De plus, on a

$$
0=\operatorname{Im} \Delta_{*}^{+}\left(Q_{2}\right)=\operatorname{Im} \Delta_{*}^{+}\left(Q_{0}\right) \subsetneq \operatorname{Im} \Delta_{*}^{+}\left(Q_{1}\right) \subsetneq \operatorname{Im} \Delta_{*}^{*}=\operatorname{Im} \Delta_{*}^{+}\left(Q_{1}\right) \oplus \operatorname{Im} \Delta_{* F_{2}}^{+} .
$$

Par suite, $\operatorname{dim} \operatorname{Im} \Delta_{*}^{+}=2$ et $\operatorname{dim} \operatorname{Im} \Delta_{*}^{+}\left(Q_{1}\right)=\operatorname{dim} \operatorname{Im} \Delta_{* F_{2}}^{+}=1$.

(iii) Le volume invariant $\bar{y}_{1} \wedge \bar{x}_{2}$ de $M$ est une classe caractéristique secondaire (non nulle) de ce sous-feuilletage, qui n'appartient pas à la sous-algèbre de $H_{D R}(M)$ engendrée par les classes caractéristiques des feuilletages de la paire.

c) Exemples de sous-feuilletages localement homogènes de la forme $\left(F_{1}, F_{2}\right)=$ $\left(F_{1}, F_{1} \cap F^{\prime}\right)$ avec $F^{\prime}$ de codimension $d=q_{2}-q_{1} \geqq 0$.

Considérons d'abord le cas suivant: Soient $F_{i}^{\prime}, i=1,2$, des feuilletages sur $M_{i}$ de codimensions $q_{1}$ et $d$ respectivement. On considère le sous-feuilletage $\left(F_{1}, F_{2}\right)=\left(F_{1}^{\prime} \times T M_{2}, F_{1}^{\prime} \times F_{2}^{\prime}\right)$ de codimension $\left(q_{1}, q_{2}\right)=\left(q_{1}, q_{1}+d\right)$ sur la variété $M=M_{1} \times M_{2}$. Alors, pour le feuilletage $F^{\prime}=T M_{1} \times F_{2}^{\prime}$ de codimension $d$ sur $M$, on a $F_{2}=F_{1} \cap F^{\prime}$. Compte tenu du Théorème 4.6 dans [5], il en résulte que l'homomorphisme caractéristique $\Delta_{*}$ de $\left(F_{1}, F_{2}\right)$ se factorise de la manière suivante :

$$
\begin{aligned}
\Delta_{*}: H\left(W O_{I}\right) & \stackrel{p_{*}}{\longrightarrow} H\left(W O_{q_{1}}\right) \otimes H\left(W O_{d}\right) \stackrel{\Delta_{*}\left(Q_{1}^{\prime}\right) \otimes \Delta_{*}\left(Q_{2}^{\prime}\right)}{\longrightarrow} \\
H_{D R}\left(M_{1}\right) \otimes H_{D R}\left(M_{2}\right) & \stackrel{k_{*}}{\cong} H_{D R}(M),
\end{aligned}
$$

où $p_{*}$ est l'homomorphisme canonique, où $\Delta_{*}\left(Q_{i}^{\prime}\right), i=1,2$, sont les homomorphismes caractéristiques des feuilletages $F_{i}^{\prime}$, et où $k_{*}$ est l'isomorphisme de Künneth (pour cela, on suppose que $\operatorname{dim} H_{D R}\left(M_{1}\right)<\infty$ ou $\operatorname{dim} H_{D R}\left(M_{2}\right)<\infty$ ). En faisant les identifications correspondantes à l'isomorphisme $k_{*}$, on obtient $\Delta_{*}\left(Q_{1}\right)$ $=\Delta_{*}\left(Q_{1}^{\prime}\right)$. De même, l'homomorphisme caractéristique $\Delta_{*}\left(Q_{0}\right)$ est donné par la composition

$$
\Delta_{*}\left(Q_{0}\right)=\Delta_{*}\left(Q_{2}^{\prime}\right) \circ p_{*}^{\prime}: H\left(W(g l(d), O(d))_{q_{2}}\right) \longrightarrow H\left(W O_{d}\right) \longrightarrow H_{D R}\left(M_{2}\right) .
$$

Par conséquent, pour $0<q_{1}<q_{2}$, les uniques classes de Godbillon-Vey de $\left(F_{1}, F_{2}\right)$ qui peuvent être non nulles sont les deux classes suivantes:

$$
\begin{aligned}
& \Delta_{*}\left[y_{1} \otimes c_{1}^{q_{1}} \otimes 1 \otimes 1\right]=\Delta_{*}\left(Q_{1}\right)\left[y_{1} \otimes c_{1}^{q_{1}}\right] \in H_{D R}\left(M_{1}\right) \subset H_{D R}(M), \\
& \Delta_{*}\left[y_{1} \otimes c_{1}^{q_{1}} \otimes y_{1}^{\prime} \otimes c_{1}^{\prime d}\right]=\Delta_{*}\left(Q_{1}\right)\left[y_{1} \otimes c_{1}^{q_{1}}\right] \otimes \Delta_{*}\left(Q_{2}^{\prime}\right)\left[y_{1}^{\prime} \otimes c_{1}^{\prime}{ }^{d}\right] \\
& \quad \in H_{D R}\left(M_{1}\right) \otimes H_{D R}\left(M_{2}\right) \cong H_{D R}(M) .
\end{aligned}
$$

En particulier, la classe de Godbillon-Vey de $F_{2}$ est nulle (cf. aussi Moussu [14]).

Supposons maintenant que les classes de Godbillon-Vey de $F_{1}^{\prime}$ et $F_{2}^{\prime}$ ne soient pas nulles. On obtient alors le résultat suivant.

THÉorème 6.4. La classe de Godbillon-Vey $\Delta_{*}\left[y_{1} \otimes c_{1}^{q_{1}} \otimes y_{1}^{\prime} \otimes c_{1}^{\prime d}\right]$ est un invariant non nul du sous-feuilletage $\left(F_{1}, F_{2}\right)$, qui ne peut pas s'obtenir en considérant séparément les feuilletages de la paire. 
Remarque. Le Théorème 6.4 est aussi valable dans le cas où $\left(F_{1}, F_{2}\right)$ est un sous-feuilletage à fibré normal orienté, avec $q_{1}$ ou $d$ impair.

D'autre part, ces résultats sont appliqués aux sous-feuilletages localement homogènes comme suit. Soient $H_{i} \subset G_{i}^{\prime} \subset \bar{G}_{i}, i=1,2$, des groupes de Lie, $h_{i} \subset$ $g_{i}^{\prime} \subset \bar{g}_{i}$ leurs algèbres de Lie. Supposons que, pour $i=1,2, H_{i}$ soit fermé dans $\bar{G}_{i}$. Soit $\Gamma_{i} \subset \bar{G}_{i}, i=1,2$, un sous-groupe discret opérant de manière proprement discontinue et sans points fixes sur $\bar{G}_{i} / H_{i}$. Considérons les feuilletages localement homogènes $F_{i}^{\prime}=F_{G_{i}^{\prime}}, i=1,2$, sur $M_{i}=\Gamma_{i} \backslash \bar{G}_{i} / H_{i}$ de codimensions $q_{1}=$ $\operatorname{dim} \bar{g}_{1} / g_{1}^{\prime}$ et $d=\operatorname{dim} \vec{g}_{2} / g_{2}^{\prime}$ respectivement. Alors, pour les groupes de Lie

$$
\bar{G}=\bar{G}_{1} \times \bar{G}_{2}, G_{1}=G_{1}^{\prime} \times \bar{G}_{2}, G_{2}=G_{1}^{\prime} \times G_{2}^{\prime}, H=H_{1} \times H_{2} \quad \text { et } \quad \Gamma=\Gamma_{1} \times \Gamma_{2},
$$

le difféomorphisme canonique $f: M=\Gamma \backslash \bar{G} / H \stackrel{\cong}{\longrightarrow} M_{1} \times M_{2}$ identifie le sous-feuilletage localement homogène $\left(F_{1}, F_{2}\right)=\left(F_{G_{1}}, F_{G_{2}}\right)$ de codimension $\left(q_{1}, q_{2}\right)=\left(q_{1}, q_{1}+d\right)$ sur $M$ avec le sous-feuilletage $\left(F_{1}^{\prime} \times T M_{2}, F_{1}^{\prime} \times F_{2}^{\prime}\right)$ de codimension $\left(q_{1}, q_{2}\right)$ sur $M_{1} \times M_{2}$. De même, le feuilletage localement homogène $F^{\prime}=F_{\bar{G}_{1} \times G_{2}^{\prime}}$ de codimension $d$ sur $M$, avec $F_{1} \cap F^{\prime}=F_{2}$, s'identifie à $T M_{1} \times F_{2}^{\prime}$. Il s'ensuit que l'homomorphisme caractéristique $\Delta_{*}$ de $\left(F_{1}, F_{2}\right)$ peut se calculer en utilisant les homomorphismes caractéristiques des feuilletages localement homogènes $F_{1}^{\prime}=F_{G_{1}^{\prime}}$ et $F_{2}^{\prime}=F_{G_{2}^{\prime}}$.

Exemple 6. Soit $\left(\bar{G}_{1}, G_{1}^{\prime}\right)$ (resp. $\left.\left(\bar{G}_{2}, G_{2}^{\prime}\right)\right)$ l'une des paires de groupes de Lie suivantes: $\left(S L\left(q_{1}+1\right), S L\left(q_{1}+1,1\right)_{0}\right),\left(S L\left(q_{1}+1\right), S L\left(q_{1}+1,1\right)\right),\left(G L^{+}\left(q_{1}+1\right)\right.$, $\left.G L^{+}\left(q_{1}+1,1\right)_{0}\right)$ et $\left(G L\left(q_{1}+1\right), G L\left(q_{1}+1,1\right)\right)$ avec $q_{1} \geqq 1$ (resp. $(S L(d+1), S L(d+1$, $\left.1)_{0}\right),(S L(d+1), S L(d+1,1)),\left(G L^{+}(d+1), G L^{+}(d+1,1)_{0}\right)$ et $(G L(d+1), G L(d+1,1))$ avec $d \geqq 1)$. Prenons $H_{1}=G_{1}^{\prime} \cap O\left(q_{1}\right)$ et $H_{2}=G_{2}^{\prime} \cap O(d)$. Pour $i=1,2$, considérons un sous-groupe discret, uniforme et sans torsion $\Gamma_{i} \subset \bar{G}_{\imath}$. Compte tenu de l'Exemple 3, on vérifie que la classe de Godbillon-Vey

$$
\Delta_{*}\left[y_{1} \otimes c_{1}^{q_{1}} \otimes y_{1}^{\prime} \otimes c_{1}^{\prime d}\right]=\left(q_{1}+1\right)^{q_{1}+1}(d+1)^{d+1} \cdot \gamma_{1 *}\left(\bar{y}_{q_{1}+1}\right) \otimes \gamma_{2 *}\left(\bar{y}_{d+1}^{\prime}\right) \neq 0 \in H_{D R}^{2 q_{2}+2}(M)
$$

du sous-feuilletage localement homegène $\left(F_{1}, F_{2}\right)=\left(F_{G_{1}}, F_{G_{2}}\right)$ de codimension $\left(q_{1}\right.$, $\left.q_{2}\right)=\left(q_{1}, q_{1}+d\right)$ sur $M=\Gamma \backslash \bar{G} / H \cong M_{1} \times M_{2}$ est un invariant (non nul) de ce sousfeuilletage, qui n'appartient pas à la sous-algèbre de $H_{D R}(M)$ engendrée par les classes caractéristiques des feuilletages de la paire.

Exemple 7. Soit $\left(\bar{G}_{1}, G_{1}^{\prime}\right)$ (resp. $\left.\left(\bar{G}_{2}, G_{2}^{\prime}\right)\right)$ l'une des paires de groupes de Lie suivantes : $\left(S U\left(q_{1}+1\right), U\left(q_{1}\right)\right)$ et $\left(U\left(q_{1}+1\right), U(1) \times U\left(q_{1}\right)\right)$ avec $q_{1} \geqq 1$ (resp. $(S U(d+1)$, $U(d))$ et $(U(d+1), U(1) \times U(d))$ avec $d \geqq 1)$. Prenons $H_{1}=\Gamma_{1}=\{e\}$ et $H_{2}=\Gamma_{2}=\{e\}$. En vertu de l'Exemple 4, la classe caractéristique secondaire

$$
\Delta_{*}\left[y_{1} \otimes c_{1}^{q_{1}} \otimes y_{1}^{\prime} \otimes c_{1}^{\prime d}\right]=\left(q_{1}+1\right)^{q_{1}+1}(d+1)^{d+1} \cdot \bar{y}_{q_{1}+1} \otimes \bar{y}_{d+1}^{\prime} \neq 0 \in H_{D R}(M)
$$

$\mathrm{du}$ sous-feuilletage homogène $\left(F_{1}, F_{2}\right)=\left(F_{G_{1}}, F_{G_{2}}\right)$ de codimension $\left(2_{q_{1}}, 2\left(q_{1}+d\right)\right)$ sur $M=\bar{G}_{1} \times \bar{G}_{2}$ est un invariant (non nul) de ce sous-feuilletage, qui n'appartient 
pas à la sous-algèbre de $H_{D R}(M)$ engendrée par les classes caractéristiques des feuilletages de la paire.

Exemple 8. Pour $q_{1} \geqq 1$ et $d \geqq 1$, considérons les cas suivants :

i) $\bar{G}_{1}=S O\left(2 q_{1}+1\right), G_{1}^{\prime}=S O\left(2 q_{1}\right), H_{1}=\Gamma_{1}=\{e\}, \bar{G}_{2}=S O(2 d+1), G_{2}^{\prime}=S O(2 d)$ et $H_{2}=\Gamma_{2}=\{e\}$.

ii) $\quad \bar{G}_{1}=S O\left(2 q_{1}+1\right), \quad G_{1}^{\prime}=S O\left(2 q_{1}\right), \quad H_{1}=\Gamma_{1}=\{e\}, \quad \bar{G}_{2}=S O(2 d), \quad G_{2}^{\prime}=S O(2 d-1)$ et $H_{2}=\Gamma_{2}=\{e\}$.

Compte tenu du Théorème 6.52 dans $[10]$, il est immédiat de voir que le sous-feuilletage homogène de codimension $\left(2 q_{1}, 2\left(q_{1}+d\right)\right)$ (resp. $\left.\left(2 q_{1}, 2\left(q_{1}+d\right)-1\right)\right)$ sur $M=S O\left(2 q_{1}+1\right) \times S O(2 d+1)$ (resp. sur $\left.M=S O\left(2 q_{1}+1\right) \times S O(2 d)\right)$ correspondant au cas i) (resp. au cas ii)) admet des invariants non nuls qui n'appartiennent pas à la sous-algèbre de $H_{D R}(M)$ engendrée par les classes caractéristiques des feuilletages de la paire. De plus, dans le cas i), on a donc $\operatorname{dim} \operatorname{Im} \Delta_{*}\left(Q_{2}\right)=$ $2^{\left[\left(\left|q_{1}-d\right|+1\right) / 2\right]}$. De même, dans le cas ii), on obtient $\operatorname{dim} \operatorname{Im} \Delta_{*}\left(Q_{2}\right)=2^{\left[\left(q_{1}-d+1\right) / 2\right]} \mathrm{si}$ $q_{1} \geqq d$ et $\operatorname{dim} \operatorname{Im} \Delta_{*}\left(Q_{2}\right)=2^{\left[\left(d-q_{1}+1\right) / 2\right]-1}$ si $q_{1}<d$.

\section{BIBLIOGRAPHIE}

[1] A. BoRel, Compact Clifford-Klein forms of symmetric spaces, Topology, 2 (1963), 111-122.

[2] R. Bотт, Lectures on characteristic classes and foliations, Lecture Notes in Math., Vol. 279, Springer, Berlin, 1972, 1-94.

[3] J.M. CARballés, Characteristic homomorphism for $\left(F_{1}, F_{2}\right)$-foliated bundles over subfoliated manifolds, Ann. Inst. Fourier, Grenoble, 33, 3 (1984), 219-245.

[4] L. A. Cordero Et X. MAsA, Characteristic classes of subfoliations, Ann. Inst. Fourier, Grenoble, 31, 2 (1981), 61-86.

[5] D. Domínguez, Sur les classes caractéristiques des sous-feuilletages, Publ. RIMS, Kyoto Univ., 23, (1987), 813-840.

[6] B.L. Feigin, Characteristic classes of flags of foliations, Funct. Anal. and its Appl., 9 (1975), 312-317.

[7] C. Godbillon et J. Vey, Un invariant des feuilletages de codimension 1, C.R. Acad. Sci. Paris, 273 (1971), 92-95.

[8] W. Greub, S. Halperin et R. Vanstone, Connections, curvature and cohomology, Academic Press, New York, Vol. I, 1972, Vol. II, 1973, Vol. III, 1976.

[9] F.W. Kamber et Ph. Tondeur, Foliated bundles and characteristic classes, Lecture Notes in Math., Vol. 493, Springer, Berlin, 1975.

[10] F. W. Kamber et Ph. Tondeur, Non-trivial characteristic invariants of homogeneous foliated bundles, Ann. Scient. École Norm. Sup., 8 (1975), 433-486.

[11] F. W. Kamber et Ph. Tondeur, On the linear independence of certain cohomology classes of $B \Gamma_{q}$, Advances in Math. Supplementary Studies, Vol. 5, Academic Press, New York, 1979, 213-263.

[12] D. Lehmann, Classes caractéristiques exotiques et $J$-connexité des espaces de connexions, Ann. Inst. Fourier, Grenoble, 24, 3 (1974), 267-306.

[13] P. Molino, Propriétés cohomologiques et propriétés topologiques des feuilletages à connexion transverse projetable, Topology, 12 (1973), 317-325. 
[14] R. Moussu, Sur les classes exotiques des feuilletages, Lecture Notes in Math., Vol. 392, Springer, Berlin, 1974, 37-42.

[15] K. Yамато, Examples of foliations with non-trivial exotic characteristic classes, Osaka J. Math., 12 (1975), 401-417.

Depaptamento de Geometría y Topología

Facultad de Matemáticas

Universidad DE SANTIAgo de Compostela

ESPAGNE 